\title{
El Niño induced changes of the coastal fish fauna off northern Chile and implications for ichthyogeography
}

Cambios de la fauna íctica del norte de Chile inducidos por El Niño y sus implicancias en la ictiogeografía

Walter Sielfeld ${ }^{1}$, Jürgen Laudien ${ }^{2}$, Mauricio Vargas $^{3}$ and Mario Villegas ${ }^{1 \dagger}$

${ }^{1}$ Universidad Arturo Prat, Av. Arturo Prat 2120, Iquique, Chile. walter.sielfeld@unap.cl

${ }^{2}$ Alfred Wegener Institute for Polar and Marine Research, P.O. Box 120161, 27515 Bremerhaven, Germany

${ }^{3}$ Servicio Nacional de Pesca, Pasaje Alessandri 470, Of. 110, Iquique, Chile

\begin{abstract}
The coastal fringe off northern Chile (18-23ㅇ) is inhabited by numerous neritic fish species of which 249 are commonly observed during normal and cold La Niña (LN) conditions. During El Niño (EN) the ichthyofauna of northern Chile changes significantly due to about 100 invading fish species normally inhabiting lower latitudes, named "septentrional invaders" and "tropic-equatorial fishes". This results in a composition including 15 fish families and 86 genera. Analyses showed that species affected by EN were not the same between the last three EN events revealing that only the epipelagic species Anchoa naso, Albula vulpes, Atherinella nocturna and Scomberesox saurus stolatus behaved consistently. Off northern Chile, EN 1982/83, 1986/87, 1991/92, 1997/98, 2002/2003 and the not clearly EN defined 2004/2005 period with their aligned thermal abnormalities of each particular event were directly related to the number of invading/affected species. Most of the alien species ( $>50 \%$ ) were epipelagic, $25 \%$

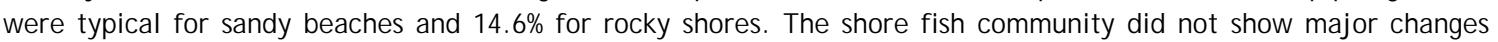
during and after EN events, as only a few invading species were recorded; just Umbrina xantii was found during all events considered. In order to understand the response of the fish community to ENSO events a biogeographical analyses of 721 fish species reported for neritic habitats (coastal epipelagic and benthic) distributed between $0^{\circ}-57^{\circ} \mathrm{S}$ (Gulf of Guayaquil to Cape Horn) was made. Their distribution ranges for normal oceanic conditions were taken from the very numereous existing literature. It is concluded that the north/south migration of Panamic and Peruvian warm water fishes during EN/ LN events along the north Chilean coast between 18-2305, indicates that it represents a wide transition zone between the Peruvian and the Chilean ichthyogeographic provinces.
\end{abstract}

Key words: ENSO, neritic fishes, northern Chile, ichthyogeography

Resumen.- La franja costera de la zona norte de Chile (18-23ㅇ) es habitada por numerosas especies de peces neríticos de las cuales 249 son comúnmente observadas durante condiciones normales y condiciones frías La Niña (LN). Durante El Niño (EN) la ictiofauna del norte de Chile experimenta cambios significativos a causa de la presencia de alrededor de 100 especies de peces invasores que durante periodos normales y/o fríos habitan en latitudes menores, siendo también nombrados "invasores septentrionales" y "peces trópico-ecuatoriales". Este conjunto incluye 15 familias y 86 géneros de peces. Los análisis mostraron que las especies afectadas por los EN no fueron las mismas durante los últimos tres eventos, revelando que solo las especies epipelágicas Anchoa naso, Albula vulpes, Atherinella nocturna y Scomberesox saurus stolatus fueron constantes. Frente al norte de Chile, durante los EN 1982/83, 1986/87, 1991/92, 1997/98, 2002/ 2003 y EN no claramente definido 2004/ 2005 y considerando las anomalías térmicas que caracterizaron a cada evento en particular, se encontró que la anomalía estuvo en relación directa con el número de especies invasoras/afectadas. Gran parte de las especies invasoras ( $>50 \%$ ) fueron epipelágicas, $25 \%$ fueron típicas de playas de arena y $14,6 \%$ de ambientes rocosos. La comunidad de peces litorales no mostró cambios mayores durante y después de los eventos EN, registrándose pocas especies invasoras; solamente Umbrina xantii fue encontrada durante todos los eventos estudiados. Con el fin de entender la respuesta de la comunidad de peces a los eventos ENSO se realizó un análisis biogeográfico de 721 especies de peces reportados para hábitats neríticos (costero, epipelágico y bentónico) distribuidas entre $0^{\circ}-57^{\circ} \mathrm{S}$ (Golfo de Guayaquil a Cabo de Hornos). La distribución latitudinal de cada especie bajo condiciones oceánicas normales se obtuvo de la numerosa literatura que existe al respecto. Se concluye que la migración norte/sur de peces de tipo panámico y peruano asociados a aguas cálidas a lo largo de la costa norte de Chile entre 18은으, representa una amplia zona de transición entre las provincias ictiogeográficas peruana y chilena.

Palabras clave: ENOS, peces neríticos, norte de Chile, ictiogeografía 


\section{INTRODUCTION}

The coastal fringe off northern Chile and southern Peru is characterized by upwelling processes, caused by friction of the S and SW winds (Burkov et al. 1971, Fuenzalida 19901선 1992, Smith 1992, Figueroa 2002, Arntz et al. 2006). Upwelling is restricted to only a narrow coastal zone (e.g., Schweigger 1960, Morales et al. 1996, Blanco et al. 2001, Hormazabal et al. 2001, Hormazabal \& Shaffer 2002) fueling the ecosystem with high levels of new production (Ryther 1969, Cushing 1971, Barber \& Smith 1981). Upwelling cells are practically permanent, showing clear seasonal variations (Fuenzalida 199011,1992) and spatial-temporal correspondence with cold (La Niña, LN) and warm (El Niño, EN) periods (Alarcón 1975, Robles et al. 1976, Rutland 1982, Bernal et al. 1983). The area surrounding Peninsula

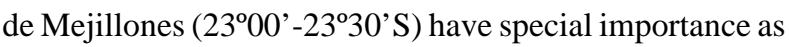
it represents the southern zoogeographical limit for several coastal warm water species (Castilla et al. 2002, Escribano et al. 2002).

Weakening of upwelling intensity and cyclic EN events of variable intensity (Quinn et al. 1978) affect species considerably (Arntz 1986, Martinez et al. 1985, Tomicic 1985, Vasquez \& Alonso-Vega 2004, Arntz et al. 2006). Barber \& Chavez (1983) found during 1982-83 EN that a reduction in primary productivity was caused by a depression of the nutricline though no reduction in the upwelling activity occurred. These events cause or allow a migration of organisms from their normal habitats to higher latitudes (Fitch 1951, Morrow 1957, Radovich 1961, Pequeño 1978, Vélez et al. 1984, Hoyos et al. 1985, Kong et al. 1985) reflected in records of several warm-water species registered only once off northern Chile (e.g., Guichenot 1848, Delfín 1899, Mann, 1954, De Buen 1959 a, b, c, Bahamonde \& Pequeño 1975, Pequeño 1989).

Bahamonde \& Pequeño (1975) and Pequeño (1997) registered around 250 common fish species (caught more than once) off northern Chile $\left(18^{\circ}-23^{\circ} \mathrm{S}\right)$. In the same region, additional 100 species were observed only during EN events (Kong et al. 1985, Sielfeld et al. 1995, Vargas et al. 1998, Vargas \& Sielfeld 1999, 1999²). These periodical ENinduced faunistic latitudinal fluctuations off northern Chile $\left(18^{\circ}-20^{\circ} \mathrm{S}\right)$ are directly related to the thermal regime. They lead to a wide and ranging transition zone between the Peruvian and Chilean zoogeographic province, as proposed by Balech (1954), López (1963), Lutjeharms (1990), Parin (1991), Pequeño \& Lamilla (1995), Ojeda et al. (2000) and Pequeño (2000).

The aim of this review is to describe EN induced changes within the fish community registered off northern Chile $\left(<23^{\circ} 40^{\prime}\right.$ 'S) between 1982 and 2005. This work represents the basis for the ongoing INCO-DEV project CENSOR and attempts to clarify whether changes of coastal fish assemblages are induced by the variability of the habitat structure (i.e., kelp beds), food availability (qualitative/ quantitative) and/or physical/chemical factors.

\section{Material AND METHODS}

The fish data used in this paper were compiled from the literature and own unpublished data. For ichthyogeographical purposes the latitudinal distribution between $0^{\circ} \mathrm{S}$ and $57^{\circ} \mathrm{S}$ (Gulf of Guayaquil to Cape Horn) of 719 neritic species normally captured at depths less than $200 \mathrm{~m}$, including epipelagic, littoral and benthic species was considered. Previous studies of the benthic fish fauna of northern Chile showed that the depth level of 100-200 m separates a neritic and a demersal assemblage (Sielfeld \& Vargas 1996, 1999), very coincident with the OMZ (Oxygen Minimum Zone) recently recognized by Fuenzalida et al. (2009) and associated with Equatorial Subsurface Water, transported from north to south along the continental slope by the Peru-Chile Undercurrent, as far south as $48^{\circ} \mathrm{S}$ (Silva \& Neshyba 1979).

"Grey" literature on littoral warm water fishes found north of $23^{\circ} 40^{\prime}$ 'S along the Chilean coast was reviewed.

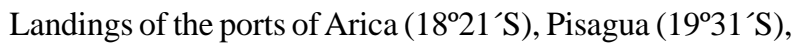
Iquique (20 $\left.20^{\circ} \mathrm{S}\right)$, Tocopilla ( $\left.20^{\circ} 05^{\prime} \mathrm{S}\right)$, Mejillones $\left(20^{\circ} 05^{\prime} \mathrm{S}\right)$

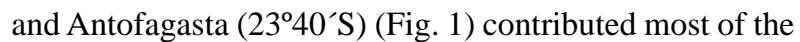
data. In addition, 20 years (1984-2004) records of the catch from several fishermen associations ("caletas de pescadores”) located between Chipana beach (2120's) and Las Machas beach $\left(18^{\circ} 10^{\prime} \mathrm{S}\right)$ were included. Furthermore, information on fishes inhabiting tide pools presented by Berrios \& Vargas $(2000,2004)$ as well as records on sublitoral fishes associated to kelp beds (Sielfeld et al. 2002) were also included in the analyses.

Additionally, historic information and results from diverse museum collections and bibliographic sources (e.g.,

${ }^{1}$ Fuenzalida R. 1990. Proceso de surgencia en la región norte de Chile, latitudes $20^{\circ} 30^{\prime} \mathrm{S}-21^{\circ} 45^{\prime} \mathrm{S}$. X J ornadas de Ciencias del Mar, Santiago, Resúmenes, p. 53.

${ }^{2}$ Vargas M \& W Sielfeld. 1999. Ictiofauna asociada al evento El Niño 1997-1998 frente a las costas de Tarapacá (I Región, Chile). XIX J ornadas de Ciencias del Mar, Iquique, Resumenes, p. 205. 


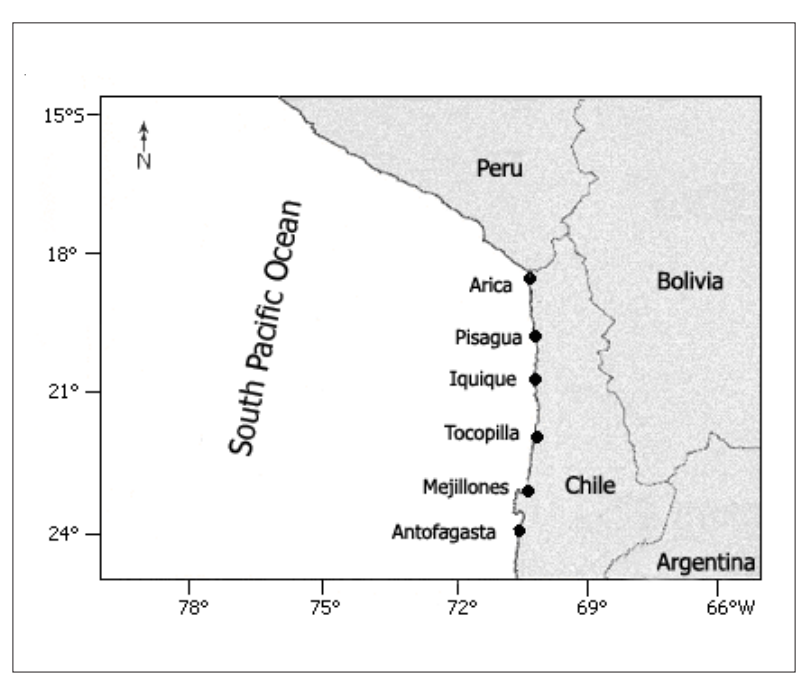

Figure 1. Main Northern Chilean ports between Arica and Antofagasta (18.21'S -2340' S), for data collect and analysis to reveal EI Niño induced changes on the coastal fish fauna / Principales puertos del norte de Chile entre Arica y Antofagasta (1821'S y 23눙'S), considerados en el análisis y obtención de datos para conocer cambios inducidos por El Niño en la fauna de los peces costeros

Guichenot 1848, Delfin 1899, 1901, Oliver-Schneider 1943a, b, Fowler 1945, De Buen 1953, 1957, 1959a, b, c, Mann 1954, Zapata \& Soto $1984^{3}$, Kong et al. 1985, Kong \& Valdés 1990, 1999), data from underwater fishing competitions, and monitoring of by-catch of the regional fisheries of Caleta de Pescadores "Guardiamarina Riquelme" between 1995 and 2003, were also considered.

In order to facilitate comparisons the listing of species (Table 1) follows Bahamonde \& Pequeño (1975), Pequeño (1989, 1997) and Chirichigno \& Vélez (1998).

Assemblages of species were determined using the hierarchical cluster analysis routine CLUSTER in the program PRIMER 6 (Clarke \& Gorley 2006) bases on the Bray-Curtis similarity measure for abundance data and Sorensen's coefficient for presence/absence data. When using CLUSTER, the permutation test SIMPROF (at 5\% level) was used to determine which clusters have significant internal structure. In the figure the later are joined by "fat” grey line.

\section{Results}

\section{Historical OVERVieW}

\section{Occasional records of uncommon fish species}

The review of the Chilean ichthyological literature revealed occasional records of fish species along the central Chilean coast apparently related with warm water events (Kong et al. 2002): the first uncommon species were Katsuwonus pelamis and Mola mola caught off Valparaiso (Guichenot 1848). The author does not provide further information; however, it is likely that the occurrence was related to a warm event. Sphyrna peruana (or S. zygaena) and Mobula tarapacana were mentioned by Philippi (1887) for northern Chile without additional data. The first species was recently (1997 and 2004) caught off Iquique and Antofagasta (details see Table 2). The second species has not been registered in the area since then. Sarda chilensis and Scomber japonicus off Valparaíso, Istiophorus audax (=Istiophorus platypterus) and Leiurus peruanus (=Seriolella violacea) off Iquique, Hemanthias peruanus with no indication of the catch locality, and Mola euryptera (=Mola ramsayi) at Chañaral are recorded by Delfín (1899) without catch date. Some of these species are regular visitors off northern Chilean waters, but the presence off central Chile is only likely during warm water events. The same holds true for Makaira marlina off Iquique cited by Bini (1952) as well as Makaira mitsukuri (=Tetrapturus audax) by Smith \& Rivas (1955, fide Kong et al. 2002).

\section{Historical records of fish related to EN events in the literature}

The following historical fish records were related to EN events by the respective authors: Mola mola registered by Philippi (1892) at Chañaral in October 1889, related to EN 1889; Pomadasys bipunctatus by Steindachner (1898) off Iquique, related to EN 1896; Pomatomus saltatrix by Delfín (1899) off Valparaíso and related to EN 1899; Rachycentron canadum reported by Delfín (1903) off Iquique, related with EN 1911; Thunnus thynnus cited by De Buen (1953) for Curaumilla: Valparaíso during EN 1953; Rhincodon typus, Sphyrna zygaena, Squatina armata, Mobula lucasana, Galeichthys peruvianus, Tylosaurus stolzmanni, Strongylura exilis, Hemiramphus saltator,

${ }^{3}$ Zapata B \& R Soto. 1984. Análisis preliminar de la ictiofauna de playas arenosas, desde Arica a Caleta Chipana, durante el período verano-otoño de 1984. Terceras J ornadas Científicas Estudiantiles, 8-9 de noviembre 1984. Universidad Arturo Prat, Iquique. (Resumen) 
Table 1. Warm-water fishes reported to be associated with warm events in the northern Chile between 1982 and 2004. (* indicates normal residents of the Peruvian province; other species are Panamic or oceanic origin) / Peces de aguas cálidas que se han reportado en asociación a eventos cálidos en el norte de Chile entre 1982 y 2004. (*indica residentes normales de la provincia Peruana; otras especies son de origen Panámico u oceánico)

\begin{tabular}{|c|c|c|c|c|c|}
\hline Family & Species & Habitat & $\begin{array}{c}\text { Southern limit of } \\
\text { extended distribution }\end{array}$ & Year & Source \\
\hline \multirow[t]{2}{*}{ Dasyatidae } & Dasyatis violacea & Epipelagic & Iquique & $1987,1988,2001,2002$ & Guzmán et al.1998; new data \\
\hline & Dasyatis brevis & Epipelagic & Antofagasta & 2000,2001 & Nelson Amado (pers. com.) \\
\hline Rhinobatidae & Rhinobatos planiceps & Soft bottom & Chipana & 1984 & Guzmán et al. 1998 \\
\hline Squatinidae & Squatina armata & Soft bottom & Chipana & 1992,2002 & Guzmán et al. 1998 \\
\hline \multirow[t]{3}{*}{ Sphyrnidae } & Sphyrna zygaena & Epipelagic & Antofagasta & $1997 / 98 / 99,2004 / 05$ & Kong et al. 1999, 2002; new \\
\hline & & & $\begin{array}{l}\text { Iquique } \\
\text { Chipana }\end{array}$ & $\begin{array}{l}1998,2004 / 05 \\
1992\end{array}$ & \\
\hline & & & Arica & $2004 / 05$ & \\
\hline \multirow{3}{*}{ Muraenidae } & Gymnothorax equatorialis & Rocky bottom & Antofagasta & $1987 / 88$ & Kong et al. 2002; new data \\
\hline & & & Iquique & 1987 & \\
\hline & Gymnothorax aff. castaneus & Rocky bottom & Chipana & 1995 & New data \\
\hline \multirow[t]{2}{*}{ Ophichthidae } & Ophichthus tetratrema $\left({ }^{*}\right)$ & Soft bottom & Arica & $1999 / 2000$ & New data \\
\hline & Ophichthus zophochir & Soft bottom & Arica & $1996 / 1997$ & New data \\
\hline Albulidae & Albula culpes & Epipelagic & Antofagasta & 1983 & Kong et al. 1985 \\
\hline \multirow[t]{2}{*}{ Engraulidae } & Anchoa nasus & Epipelagic & Arica & 2000 & Vargas \& Peredo $2001 b^{5}$ \\
\hline & Anchoa naso & Epipelagic & $\begin{array}{l}\text { Chipana } \\
\text { Iquique }\end{array}$ & $\begin{array}{l}1984,1988,1989 \\
1991,1999\end{array}$ & $\begin{array}{l}\text { Guzmán et al. 1998, Sielfeld et } \\
\text { al. } 1999\end{array}$ \\
\hline \multirow[t]{6}{*}{ Clupeidae } & Etrumeus teres & Epipelagic & Iquique & $1983,1992,1994$ & $\begin{array}{l}\text { Kong et al. 1985, 2002, } \\
\text { Guzmán et al. } 1998\end{array}$ \\
\hline & & & Arica & $\begin{array}{l}1989 \\
1989\end{array}$ & \\
\hline & & & Antofagasta & $1998 / 99$ & \\
\hline & Opisthonema libertate & Epipelagic & Iquique & 1983 & Kong et al. 1985, 1999, 2002, \\
\hline & & & Antofagasta & $1984,1998 / 99$ & Guzmán et al. 1998 \\
\hline & & & Iquique & 1998 & \\
\hline \multirow[t]{2}{*}{ Synodontidae } & Synodus lacertimus & Not defined & Iquique & 1984 & $\begin{array}{l}\text { Kong et al. 1985, Guzmán et } \\
\text { al. } 1998\end{array}$ \\
\hline & Synodus scituliceps & Not defined & Antofagasta & 1985,1997 & Kong et al. 2002 \\
\hline Atherinidae & Atherinella nocturna & Epipelagic & $\begin{array}{l}\text { Arica, Pisagua, Iquique } \\
\text { Chipana }\end{array}$ & $\begin{array}{l}1984,1999 \\
1984,1990,1991,1997\end{array}$ & $\begin{array}{l}\text { Kong et al. 1985, Guzmán et } \\
\text { al. 1998, Sielfeld et al. 1999, } \\
\text { new data }\end{array}$ \\
\hline Fistularidae & Fistularia commersoni & Epipelagic & Iquique & 1998 & Guzmán et al. 1998 \\
\hline \multirow[t]{7}{*}{ Exocoetidae } & Cypselurus heterurus & Epipelagic & $\begin{array}{l}\text { Antofagasta } \\
\text { Chipana }\end{array}$ & $\begin{array}{l}1983 \\
1983,1994\end{array}$ & $\begin{array}{l}\text { Kong et al. 1985, Guzmán et } \\
\text { al. } 1998\end{array}$ \\
\hline & Fodiator acutus & Epipelagic & Iquique & 1992 & Guzmán et al. 1998, Kong et al. \\
\hline & & & Chipana & 1997/98 & \\
\hline & & & Antofagasta & 1998/99 & \\
\hline & Hirundichthys rondeletti & Epipelagic & Pta. Madrid & 1993 & Guzman et al. 1998 ; new data \\
\hline & & & Chucumata & 1993 & \\
\hline & & & Chipana & 1994 & \\
\hline Hemiramphidae & Hemiramphus saltador & Epipelagic & Antofagasta & $1983 / 84$ & Kong et al. 1985 \\
\hline \multirow[t]{3}{*}{ Belonidae } & Strongylura exilis & Epipelagic & $\begin{array}{l}\text { Caleta Errázuriz } \\
\text { Antofagasta, Iquique }\end{array}$ & $\begin{array}{l}1983 \\
1984,1997,1998 / 99\end{array}$ & $\begin{array}{l}\text { Kong et al. } 1985,1999 \text {, new } \\
\text { data }\end{array}$ \\
\hline & Belone stolzmanni & Epipelagic & Iquique & 1984,1999 & Guzmán et al. 1998, Sielfeld et \\
\hline & & & Chipana & 1998 & al. 1999 \\
\hline Scomberesocidae & Scomberesox saurus & Epipelagic & Mejillones & 1984 & Kong et al. 1985, Guzmán et \\
\hline \multirow{4}{*}{ Trachypteridae } & & & P & 1083 & Kong $e t a l 1985$ \\
\hline & Trachypterus altivelis & Epipelagic & $\begin{array}{l}\text { Antofagasta } \\
\text { Iquique }\end{array}$ & $\begin{array}{l}1983 \\
1984\end{array}$ & Kong et al. 1985 \\
\hline & Zu cristatus & Epipelagic & Iquique & 1987 & Guzmán et al. 1998 \\
\hline & & & Punta Gruesa & 1992 & \\
\hline \multirow[t]{2}{*}{ Syngnathidae } & Hippocampus ingens $\left({ }^{*}\right)$ & Not defined & $\begin{array}{l}\text { Tocopilla } \\
\text { Iquique }\end{array}$ & $\begin{array}{l}1983 \\
1998\end{array}$ & $\begin{array}{l}\text { Kong et al. 1985, 1999, 2002, } \\
\text { new data }\end{array}$ \\
\hline & & & Antofagasta & $1998 / 99$ & \\
\hline \multirow[t]{5}{*}{ Scorpaenidae } & Prionotus stephanophrys & Epipelagic & Antofagasta & $1982,1998 / 99$ & Kong et al. 1985,2002 \\
\hline & & & Mejillones & 1983 & Guzmán et al., 1998, new data \\
\hline & & & Iquique & 1983,1992 & \\
\hline & Prionotus quiescens & Epipelagic & Antofagasta, & $1998 / 99$ & Kong et al. 1999, 2002 \\
\hline & Scorpaena hystrio $\left({ }^{*}\right)$ & Rocky bottom & Iquique & 1996,1999 & Sielfeld \& Vargas 2000 \\
\hline
\end{tabular}


Table 1 continued / Continuación Tabla 1

\begin{tabular}{|c|c|c|c|c|c|}
\hline Family & Species & Habitat & $\begin{array}{l}\text { Southern limit of } \\
\text { extended distribution }\end{array}$ & Year & Source \\
\hline Priacanthidae & Pristigenys serrula $\left({ }^{*}\right)$ & Rocky bottom & $\begin{array}{l}\text { Iquique } \\
\text { Antofagasta }\end{array}$ & $\begin{array}{l}1993 \\
1998 / 99\end{array}$ & Kong et al. 1999 , new data \\
\hline Antennariidae & Antennarius avalonis & Rocky bottom & Iquique & 2000 & New data \\
\hline \multirow[t]{2}{*}{ Ariidae } & Galeichthys periviamus (*) & Soft bottom & $\begin{array}{l}\text { Mejillones Antofagasta } \\
\text { Iquique }\end{array}$ & $\begin{array}{l}1983 \\
1995\end{array}$ & $\begin{array}{l}\text { Kong et al. 1985, Guzmán et } \\
\text { al. } 1998\end{array}$ \\
\hline & Arius seemanni & Soft bottom & Arica & 2000 & Vargas \& Peredo $2001 b^{5}$ \\
\hline Prolatilidae & Caulolatilus princeps $\left({ }^{*}\right)$ & Soft bottom & $\begin{array}{l}\text { Iquique } \\
\text { Pisagua }\end{array}$ & $\begin{array}{l}1992 \\
2002\end{array}$ & Guzmán et al. 1998, new data \\
\hline Cliniidae & Labrisomus xantii & Rocky bottom & Caleta Buena & 1999 & New data \\
\hline Coryphaenidae & Coryphaena hippurus & Epipelagic & Antofagasta & $1983,1998 / 99$ & Kong et al. 1985, 1999 \\
\hline \multirow[t]{9}{*}{ Carangidae } & Caranx caninus & Epipelagic & Iquique & 1984,1998 & $\begin{array}{l}\text { Guzmán et al. 1998, Vargas \& } \\
\text { Sielfeld } 1999\end{array}$ \\
\hline & $\begin{array}{l}\text { Caranx caballus } \\
\text { Pseudocaranx dentex } \\
\text { Decapterus macrosoma }\end{array}$ & $\begin{array}{l}\text { Epipelagic } \\
\text { Epipelagic } \\
\text { Epipelagic }\end{array}$ & $\begin{array}{l}\text { Antofagasta } \\
\text { Iquique } \\
\text { Antofagasta } \\
\text { Arica }\end{array}$ & $\begin{array}{l}1983 / 84,1998 / 99 \\
1998 \\
1984,1998 / 99 \\
2000\end{array}$ & $\begin{array}{l}\text { Kong et al. 1985, 1999, } 2002 \\
\text { New data } \\
\text { Kong et al. 1985, 1999, 2002, } \\
\text { Vargas \& Peredo 2001 b }\end{array}$ \\
\hline & Oligoplites refulgens & Epipelagic & Antofagasta & $1998 / 99$ & Kong et al. 1999, 2002 \\
\hline & Trachinotus paitensis $\left({ }^{*}\right)$ & Epipelagic coastal & $\begin{array}{l}\text { Mejillones Antofagasta } \\
\text { Arica, Iquique } \\
\text { Chipana }\end{array}$ & $\begin{array}{l}1983 \\
1984 \\
1990,1992\end{array}$ & $\begin{array}{l}\text { Kong et al. 1985, Guzmán et } \\
\text { al. } 1998\end{array}$ \\
\hline & Naucrates ductor & Epipelagic & $\begin{array}{l}\text { Pisagua } \\
\text { Iquique }\end{array}$ & $\begin{array}{l}1984 \\
1992,1997,1998\end{array}$ & $\begin{array}{l}\text { Guzmán et al. } 1998 \text {, new data } \\
\text { Vargas \& Sielfeld, } 1999\end{array}$ \\
\hline & Seriola peruana & Epipelagic & $\begin{array}{l}\text { Antofagasta } \\
\text { Iquique }\end{array}$ & $\begin{array}{l}1983,1998 / 99 \\
1992,1997\end{array}$ & $\begin{array}{l}\text { Kong et al. } 1985 \text {, Kong et al. } \\
2002 \text {, new data }\end{array}$ \\
\hline & Alectis ciliaris & Epipelagic & $\begin{array}{l}\text { Iquique, Mejillones } \\
\text { Antofagasta } \\
\text { Iquique }\end{array}$ & $\begin{array}{l}1983,1998 / 99 \\
1984\end{array}$ & $\begin{array}{l}\text { Kong et al. 1985, 1999, 2002, } \\
\text { Guzmán et al. } 1998\end{array}$ \\
\hline & Selene periviana & Epipelagic & $\begin{array}{l}\text { Iquique, Mejillones } \\
\text { Antofagasta } \\
\text { Iquique }\end{array}$ & $\begin{array}{l}1983 \\
1992\end{array}$ & $\begin{array}{l}\text { Kong et al. 1985, Guzmán et } \\
\text { al. } 1998\end{array}$ \\
\hline & Selene brevoorti & Epipelagic & $\begin{array}{l}\text { Mejillones Antofagasta } \\
\text { Iquique } \\
\text { Antofagasta }\end{array}$ & $\begin{array}{l}1983 \\
1984,1998 \\
1998 / 99\end{array}$ & $\begin{array}{l}\text { Kong et al. } 1985,1999,2002, \\
\text { Guzmán et al. } 1998\end{array}$ \\
\hline Nematistidae & Nematistius pectoralis & Epipelagic & Sur de Taltal & 1992 & Kong et al. 2002 \\
\hline Chaetodontidae & Chaetodon humeralis & Rocky bottom & $\begin{array}{l}\text { Iquique } \\
\text { Antofagasta }\end{array}$ & $\begin{array}{l}1984 \\
1998 / 99\end{array}$ & Kong et al. 1985, 1999, 2002 \\
\hline Echeneidae & Rhombochirus osteochir & Epipelagic & Frente río Loa & 1998 & Vargas \& Sielfeld $1999^{2}$ \\
\hline Sparidae & Calamus brachysomus & Soft bottom & $\begin{array}{l}\text { Frente río Loa } \\
\text { Arica } \\
\text { Antofagasta }\end{array}$ & $\begin{array}{l}1998 \\
2000 \\
1998 / 99\end{array}$ & $\begin{array}{l}\text { Vargas \& Sielfeld } 1999^{2}, \\
\text { Vargas \& Peredo } 2001 b^{3}, \text { Kong } \\
\text { et al. } 1999\end{array}$ \\
\hline Gerridae & Eucinostomus argenteus & Soft bottom & $\begin{array}{l}\text { Caleta Errázuriz } \\
\text { Iquique } \\
\text { Antofagasta }\end{array}$ & $\begin{array}{l}1983 \\
1985,1999 \\
1998 / 99\end{array}$ & $\begin{array}{l}\text { Kong et al. } 1985 \text { 2002, } \\
\text { Guzmán et al. 1998, Sielfeld et } \\
\text { al. } 1999\end{array}$ \\
\hline Bramidae & $\begin{array}{l}\text { Taractes rubescens } \\
\text { Brama brama }\end{array}$ & $\begin{array}{l}\text { Epipelagic } \\
\text { Epipelagic }\end{array}$ & $\begin{array}{l}\text { Iquique } \\
\text { Mejillones } \\
\text { Antofagasta }\end{array}$ & $\begin{array}{l}1984 \\
1984 \\
1983\end{array}$ & $\begin{array}{l}\text { Kong et al. } 1985 \\
\text { Kong et al. } 1985\end{array}$ \\
\hline \multirow[t]{5}{*}{ Sciaenidae } & $\begin{array}{l}\text { Larimus pacificus }\left({ }^{*}\right) \\
\text { Paralonchurus dumerilii }\left({ }^{*}\right) \\
\text { Paralonchurus peruamus }\left({ }^{*}\right) \\
\text { Umbrina xantii }\end{array}$ & $\begin{array}{l}\text { Soft bottom } \\
\text { Soft bottom } \\
\text { Soft bottom } \\
\text { Soft bottom }\end{array}$ & $\begin{array}{l}\text { Iquique } \\
\text { Iquique } \\
\text { Arica } \\
\text { Iquique } \\
\text { Chipana } \\
\text { Antofagasta }\end{array}$ & $\begin{array}{l}1983 \\
1983 \\
1985,1993 \\
1983,1999 \\
1984,1989 \\
1998 / 99\end{array}$ & $\begin{array}{l}\text { Kong et al. } 1985 \\
\text { Kong et al. } 1985 \\
\text { Guzmán et al. } 1998, \text { new data } \\
\text { Kong et al. } 1985,2002, \\
\text { Guzmán et al. } 1998, \text { Sielfeld et } \\
\text { al. } 1999\end{array}$ \\
\hline & Stellifer minor $\left(^{*}\right)$ & Soft bottom & Arica & 1999,2000 & $\begin{array}{l}\text { Vargas \& Sielfeld } 1999 b^{2} \text {, new } \\
\text { data }\end{array}$ \\
\hline & Stellifer erycimba $\left({ }^{*}\right)$ & Soft bottom & Iquique & 1999 & Sielfeld et al. 1999 \\
\hline & Sciaena starksii $\left({ }^{\circ}\right)$ & Soft bottom & Arica & 2000 & Vargas \& Peredo 2001a ${ }^{4}$ \\
\hline & Menticirrhus undulatus & Soft bottom & Arica & 2000 & Vargas \& Peredo 2001a ${ }^{4}$ \\
\hline Scaridae & Nicholsina denticulatum & Rocky bottom & Iquique & 2000 & new data \\
\hline Kyphosidae & Kyphosus analogus & Rocky bottom & Antofagasta & 1983 & Kong et al. 1985 \\
\hline \multirow[t]{2}{*}{ Mullidae } & Pseudupeneus grandisquamis & Soft bottom & $\begin{array}{l}\text { Iquique, Mejillones } \\
\text { Antofagasta } \\
\text { Iquique }\end{array}$ & $\begin{array}{l}1983 \\
1983,1984,1998 / 99 \\
1984,1999\end{array}$ & $\begin{array}{l}\text { Kong et al. 1999, 2002, } \\
\text { Guzmán et al. 1998, Sielfeld et } \\
\text { al. } 1999\end{array}$ \\
\hline & Mulloidichthys dentatus & Soft bottom & Arica & 2000 & Vargas \& Peredo 2001 $\mathrm{b}^{5}$ \\
\hline
\end{tabular}


Table 1 continued / Continuación Tabla 1

\begin{tabular}{|c|c|c|c|c|c|}
\hline Family & Species & Habitat & $\begin{array}{l}\text { Southern limit of } \\
\text { extended distribution }\end{array}$ & Year & Source \\
\hline \multirow[t]{3}{*}{ Labridae } & Bodianus eclancheri & Rocky bottom & Iquique & 1987 & Vargas et al. 1998 \\
\hline & Halichoeres dispilus $\left({ }^{*}\right)$ & Rocky bottom & Arica & 1987 & Vargas et al. 1998, Sielfeld et \\
\hline & & & Chucumata & $1987,2000,2001$ & al. 2002 \\
\hline Serranidae & Epinephelus itajara & Not defined & Antofagasta & 1997/98 & $\begin{array}{l}\text { Kong et al. 1999; Rojas \& } \\
\text { Pequeño 2001 }\end{array}$ \\
\hline \multirow[t]{4}{*}{ Polynemidae } & Polydactylus approximans & Soft bottom & Iquique, Tocopilla, & 1983 & $\begin{array}{l}\text { Kong et al. 1985, 1999, 2002, } \\
\text { Gurmán et al. } 1998\end{array}$ \\
\hline & & & Punta Arenas & $1998 / 99$ & \\
\hline & & & Antofagasta & & \\
\hline & Polydactylus opercularis & Soft bottom & Iquique, Mejillones & 1983 & Kong et al. 1985 \\
\hline \multirow[t]{3}{*}{ Sphyraenidae } & Sphyraena ensis & Epipelagic & $\begin{array}{l}\text { Iquique, Mejillones, } \\
\text { Antofagasta }\end{array}$ & $1983,1998 / 99$ & Kong et al. 1985, 1999, 2002 \\
\hline & Sphyraena idiasthes & Epipelagic & Iquique & 1983,1998 & Kong et al. 1985, Vargas \& \\
\hline & & & Antofagasta & 1984 & Sielfeld 1999 \\
\hline \multirow{12}{*}{ Scombridae } & Atxis thazard & Epipelagic & Mejillones, Antofagasta & $1983,1998 / 99$ & Kong et al. 1998, 2002 \\
\hline & Auxis rochei & Epipelagic & Iquique & 1989,1993 & Guzmán et al. 1998, Kong et \\
\hline & & & Junin & 1993 & al. 1999,2002 \\
\hline & & & Punta Madrid & 1992 & \\
\hline & & & Antofagasta & $1985,1998 / 99$ & \\
\hline & Katsuwonus pelamis & Epipelagic & Antofagasta & 1984 & Kong et al. 1985, Guzmán et \\
\hline & & & Caleta Lautaro & 1993 & al. 1998 \\
\hline & Sarda chilensis $\left({ }^{*}\right)$ & Epipelagic & Antofagasta & 1983 & Kong et al. 1985, Guzmán et \\
\hline & & & Iquique & 1992 & 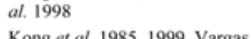 \\
\hline & Scomberomorus sierra & Epipelagic & Antofagasta & $1983,1997 / 98$ & Kong et al. 1985, 1999, Vargas \\
\hline & & & Chipana & 1989 & $\begin{array}{l}\text { \& Peredo } 2001 \mathrm{~b}^{5} \text {, Guzmán et } \\
\text { al. } 1998\end{array}$ \\
\hline & & & Arica & 2000 & al. 1998 \\
\hline \multirow[t]{3}{*}{ Stromateidae } & Psenes sio & Epipelagic & Iquique & 1984,1986 & $\begin{array}{l}\text { Kong et al. 1985, Guzmán et } \\
\text { al. } 1998\end{array}$ \\
\hline & Peprilus medius & Epipelagic & Chipana & 1992 & Sielfeld et al. 1995, Kong et al. \\
\hline & & & Antofagasta & 1997/98 & 1999,2000 \\
\hline \multirow{2}{*}{ Centrolophidae } & Schedophilus medusophaghus & Epipelagic & Frente río Loa & 1992 & Guzmán et al. 1998 \\
\hline & Centrolophus niger & Epipelagic & Punta Paquica & 1992 & Guzmán et al. 1998 \\
\hline \multirow[t]{2}{*}{ Nomeidae } & Cubiceps carinatus & Epipelagic & Patache & 1989 & Guzmán et al. 1998 \\
\hline & Cubiceps caeruleus & Epipelagic & $\begin{array}{l}\text { Iquique, Mejillones, } \\
\text { Antofagasta }\end{array}$ & 1983 & Kong et al. 1985 \\
\hline Tetragonuridae & Tetragonurus cuvieri & Epipelagic & Mejillones & 1984 & Kong et al. 1985 \\
\hline \multirow[t]{3}{*}{ Bothidae } & Bothus constellatus & Soft bottom & Caleta Buena & 1998 & Sielfeld $e t$ al. 2003 \\
\hline & Etropus ectenes & Soft bottom & Iquique & 1999 & Sielfeld et al. 2003 \\
\hline & Achirus klunzingeri & Soft bottom & Antofagasta & 1984 & Sielfeld et al. 2003 \\
\hline Symphuridae & Symphurus elongatus & Soft bottom & Arica & 2000 & Sielfeld et al. 2003 \\
\hline \multirow[t]{5}{*}{ Balistidae } & Aluterus monocerus & Rocky bottom & Mejillones & 1983 & Kong 1985 \\
\hline & & & Iquique & 1984 & \\
\hline & Balistes polylepis & Rocky bottom & Antofagasta & 1983 & Kong et al. 1985 , new data \\
\hline & & & Iquique & $1984,2002,2004$ & \\
\hline & Pseudobalistes naufragium & Rocky bottom & Antofagasta & 1983 & Kong et al. 1985 \\
\hline \multirow[t]{5}{*}{ Tetraodontidae } & Sphoeroides lobatus & Rocky bottom & Caleta Errázuriz & 1983 & Kong et al. 1985, 1999, 2002 \\
\hline & & & Iquique & 1989 & Guzmán et al. 1998 \\
\hline & & & Antofagasta & 1998/99 & \\
\hline & Sphoeroides trichocephalus & Rocky bottom & Iquique & 1989 & Guzmán et al. 1998, Kong et \\
\hline & & & Antofagasta & 1998/99 & al. 2002 \\
\hline \multirow[t]{3}{*}{ Diodontidae } & Chilomycterus affinis & Epipelagic & Iquique & 1990 & Sielfeld \& Vargas 2000 \\
\hline & & & Mejillones del Norte & 1998 & \\
\hline & Diodon hystrix & Epipelagic & Iquique & 1984 & Kong et al. 1985 \\
\hline \multirow[t]{4}{*}{ Molidae } & Mola mola/ramsayi & Epipelagic & Antofagasta & $\begin{array}{l}1983,1985, \\
1998 / 99,1984\end{array}$ & $\begin{array}{l}\text { Kong et al. 1985, 2002, } \\
\text { Guzmán et al. } 1998\end{array}$ \\
\hline & & & Iquique & 1995 & \\
\hline & Masturus lanceolatus & Epipelagic & Antofagasta & 1985 & Kong et al. 2002, Guzmán et \\
\hline & & & Iquique & 1985 & al. 1998 \\
\hline
\end{tabular}


Fodiator acutus, Rachycentron canadum, Makaira audax, Neothunus macropterus, Mupus peruanus, Echeneis brachyptera and Balistes polylepis reported by De Buen (1957) in relation with EN 1957; Mola ramsayi mentioned by Oliver-Schneider (1930) for Coliumo, related to EN 1926/27; Stellifer erycimba and Sphyraena ensis registered by Ojeda (1978) for EN 1978; Mola ramsayi listed by Bahamonde (1963) off Isla Grande de Chiloé, Pristigenys serrula reported by Alberti (1963) off Antofagasta, Cheilopogon heterurus, Prionotus stephanophrys, Sphyraena ensis and Scomberomorus sierra reported by Kong et al. (1981), Etropus ectenes, Achirus klunzingeri and Symphurus elongatus by Sielfeld et al. (2003), Alectis ciliaris, Selene peruviana and Pseudupeneus grandisquamis mentioned by Martinez et al. (1983) and Chilomycterus affinis listed by Sielfeld \& Vargas (2000), all for northern Chile, and Arius seemanni, Sciaena starksii and Menticirrhus undulatus landed at the harbour of Arica (Vargas \& Peredo 2001a4, b $\mathrm{b}^{5}$ ).

\section{Species lists of Chilean fishes}

Several species lists of Chilean fishes (Fowler 1945, Bahamonde \& Pequeño 1975, Pequeño 1989, 1997) include additional warm-water species, collected only once in Chilean waters and the occurrence was probably be related to warm events. Between these, the following are Chromis intercrusma reported by Hildebrand (1946) as "probable" species at the Juan Fernández Islands, and classified as "septentrional invader" by Mann (1954) reaching waters south of Coquimbo (central Chile). Prionodes huascari (=Serranus huascari) was recorded by Steindachner (1902) and again classified as "septentrional invader" by Mann (1954). This species may as well reach areas south of Coquimbo, Hemianthias peruanus reported by Jordan \& Evermann (1898) for Tarapacá (northern Chile), unfortunately the authors don't provide the sampling locality, also not specified by Jordan \& Eigenmann (1890) and Delfín (1901) for the same species. Alfestes afer (probably = Alphestes immaculatus registered by Hooker (1998) for Peru) was registered by Fowler (1945) for Chile, without locality and date. Pinguilabrum punctatum (=Graus nigra) listed by Chirichigno (1974) between Casma and Antofagasta (northern Chile), without mentioning sampling locality and date, is typical of the central coast of Chile and very rare north of $14^{\circ} \mathrm{S}$ (Vargas
\& Pequeño 2004). The same holds true for Diplectrum conceptione for which the author indicated a geographical range from Ecuador to Talcahuano (central Chile). Bodianus diplotaenia was registered by Schmeltz (1869, 1874 fide Fowler 1945) for Chile without stating the collection locality. Peprilus medius was found by Sielfeld et al. (1995) off Iquique, and Bodianus eclancheri and Halichoeres dispilus were reported by Vargas et al. (1998) off Iquique the first, and off Arica and Iquique, the second (northern Chile).

\section{RECORDS OF FISHES RELATED TO WARM WATER EVENTS}

\section{General considerations}

A relationship of alien fishes to EN events was first established by Mann (1954). He found two categories and proposed the term "stenotherm fishes of warm waters" for species regularly inhabiting subtropical waters around the Chilean oceanic islands and "septentrional invaders of the Peru Current" for fishes restricted to the Peruvian coast during cold events and expanding their distribution during the warm phase of the ENSO.

\section{Species list}

Since EN 1982-83, a systematic record of fish species from tropical waters begun, Kong et al. (1985) and Kong \& Bolados (1987) reported 51 species for northern Chile. Guzmán et al. (1998) expanded the list for northern Chile since 1987. Samples were archived in the zoological collection of the Universidad Arturo Prat, Iquique.

Furthermore, information on warm-water fish species caught during EN events off northern Chile between 1982 and 2002 have been presented by Kong et al. (1985), Sielfeld et al. (1995) and Vargas et al. (1998) and summarized in Table 2. This list includes 96 species (5 chondrycthians and 91 teleosteans) corresponding to 49 families. For eight of these species the present review is the first record for the Chilean coast (Gymnothorax aff. equatorialis, Ophictus tetratrema, O. zophochir, Labrisomus xantii, Caranx georgianus, Nicholsina denticulata, Hirundichthys rondeletii, Antennarius avalonis) (Table 2). Species normally distributed during summer of non-EN years north of Antofagasta $\left(<23^{\circ}\right.$ S, Fig. 1) like Sardinops sagax and Scomber japonicus, but which extend their distribution to the south during EN events (Kong et al. 1985) have been

\footnotetext{
${ }^{4}$ Vargas M \& R Peredo. 2001a. Primer registro en Chile de Sciaena starksi y Menticirrhus undulatus (Pisces, Sciaenidae) y su interes para la pesca artesanal de la I Región. XXI Congreso de Ciencias del Mar, Viña del Mar, Resúmenes, p. 106.

${ }^{5}$ Vargas M \& R. Peredo. 2001b. Nuevos registros de peces en las costas de Arica (I Región, Chile) durante el período 2000 - 2001, y su relación con eventos EI Niño. XXI Congreso de Ciencias del Mar, Viña del Mar, Resúmenes, p. 106.
} 
Table 2. Fish species associated with warm-water events (grey) and recorded between 1982 and 2004 off northern Chile (Shadding and frame indicate the different warm events and after-event subsisting warm water species) / Especies de peces asociados a eventos cálidos (gris) y registrados entre 1982 y 2004 frente al norte de Chile (el sombreado y marco indica los diferentes eventos cálidos y la subsistencia de las especies de aguas cálidas post-evento

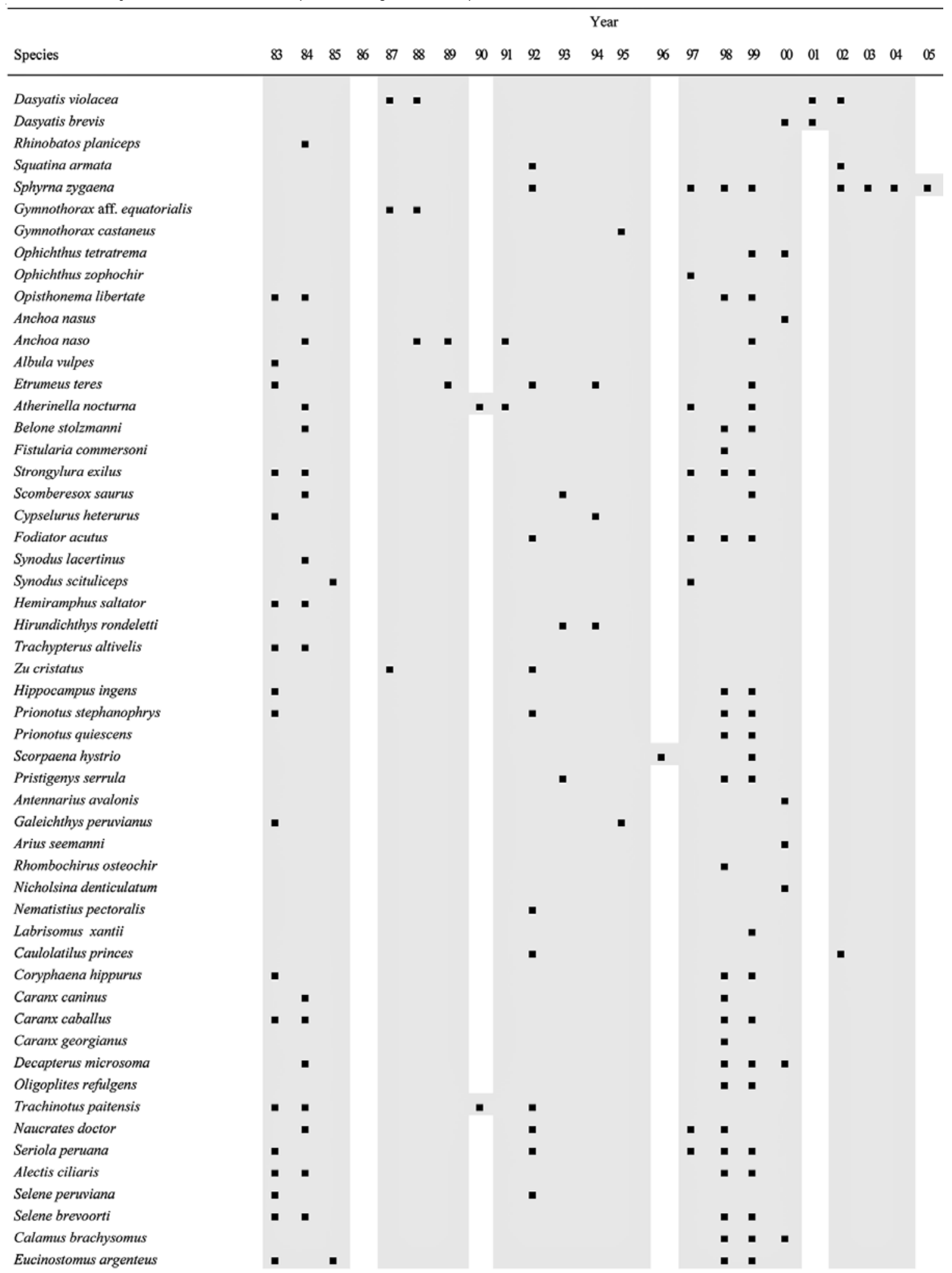

712 Sielfeld et al.

EN induced changes on fish fauna of northern Chile 
Table 2 continued / Continuación Tabla 2

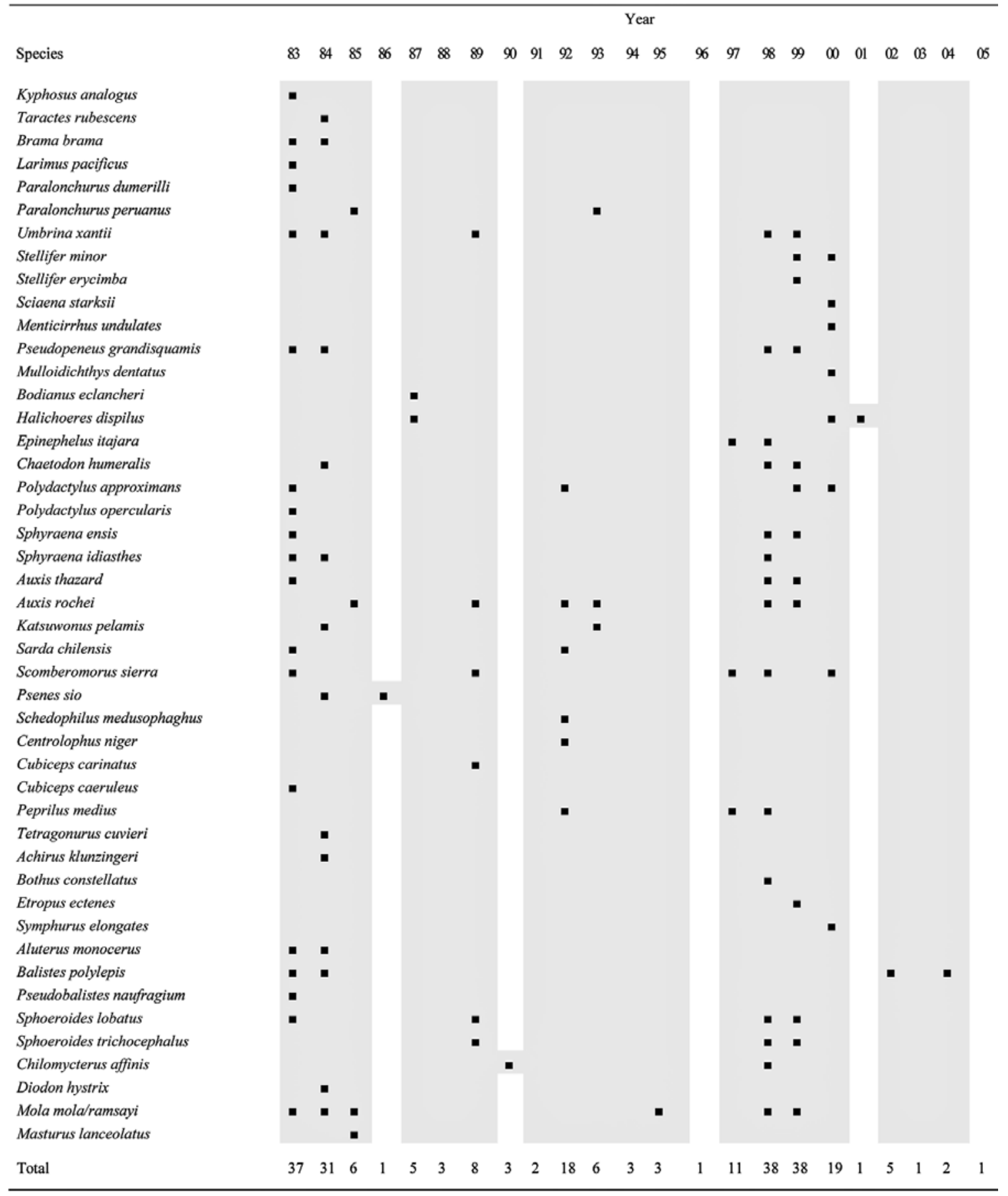


excluded from Table 2. They may be considered as EN visitors for central Chile, but normal for northern Chile.

\section{Habitat preference of warm-water species}

Fishes occurring during EN events mainly mobile epipelagic species (55 species: 55\%; Table 3) probably migrating with the water masses. Littoral species (25) include fishes typically inhabiting sandy beaches and soft bottom habitats mainly represented by the family Sciaenidae, are the second dominant group (Vargas 1993), while 16 species associated with rocky shores were registered (Sielfeld et al. 2002.) (Table 3). This tend is valid for all warm events analysed, where epipelagic and soft bottom migrating fishes always were the most frequent (Table 3).

\section{North-south gradient in species number of invading fishes}

Table 4 lists the number of southwards invading Panamic and Peruvian fish species and families found at different locations (see also Fig. 1) along the northern Chilean coast during warm water events. A clear north-south gradient is obvious (Fig. 2) supporting the transition character of the study area between the Chilean and Peruvian zooprovinces (sensu Balech 1954), already emphasized by Sielfeld \& Vargas (1996) for engybenthic fish assemblages (sensu

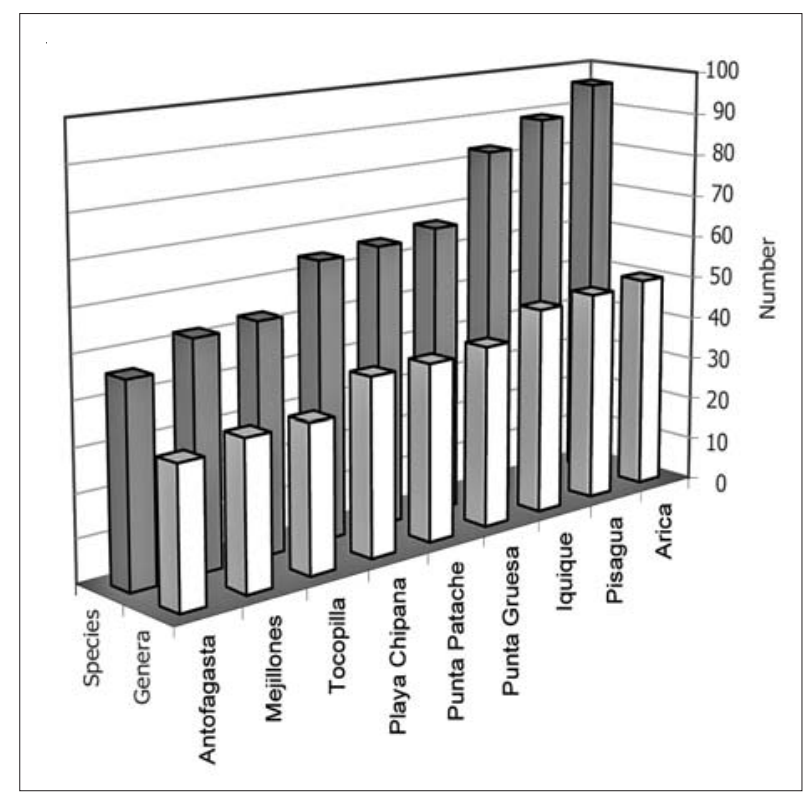

Figure 2. North south gradient in the number of warm-water fishes recorded along the northern Chilean coast / Gradiente norte sur en el número de peces de aguas cálidas registrados a lo largo de la costa chilena del norte
Table 3. Fishes associated to EN events in northern Chile and its habitat preference / Peces asociados a eventos EN en el norte de Chile y sus preferencias de habitat

\begin{tabular}{|c|c|c|c|c|c|c|c|c|c|c|}
\hline \multirow[b]{3}{*}{ EN events } & \multicolumn{8}{|c|}{ Habitat type } & & \\
\hline & \multicolumn{2}{|c|}{ Epipelagic } & \multicolumn{2}{|c|}{ Soft bottom } & \multicolumn{2}{|c|}{ Rocky shore } & \multicolumn{2}{|c|}{ Not defined } & \multicolumn{2}{|c|}{ TOTAL } \\
\hline & $\mathrm{N}$ & $\%$ & $\mathrm{~N}$ & $\%$ & $\mathrm{~N}$ & $\%$ & $\mathrm{~N}$ & $\%$ & $\mathrm{~N}$ & $\%$ \\
\hline $82 / 85$ & 38 & 66.6 & 12 & 21.1 & 6 & 10.5 & 1 & 1.8 & 57 & 100 \\
\hline $87 / 89$ & 9 & 56.3 & 1 & 6.3 & 5 & 31.3 & 1 & 6.3 & 16 & 100 \\
\hline $91 / 95$ & 22 & 73.3 & 5 & 16.7 & 3 & 10.0 & 0 & 0.0 & 30 & 100 \\
\hline $97 / 98$ & 34 & 54.8 & 16 & 25.8 & 9 & 14.5 & 3 & 4.8 & 62 & 100 \\
\hline $02 / 04$ & 2 & 40.0 & 2 & 40.0 & 1 & 20.0 & 0 & 0.0 & 5 & 100 \\
\hline All events & 55 & 55.0 & 25 & 25.0 & 16 & 16.0 & 4 & 4.0 & 100 & 100 \\
\hline
\end{tabular}

Mead 1970) for fishes living not demersal, but up to 20 metres above the bottom.

\section{Relation with thermal anomaly degree}

Table 5 shows that the intensity of the thermal anomaly is related to the number of invading warm-water fish species off northern Chile. Species involved vary between events. Only the following epipelagic species Anchoa naso, Albula vulpes, Atherinella nocturna and Scomberesox saurus were found consistently during the last three EN events. Nearshore fish communities were affected by EN to a much lesser degree, resulting from only a few invading species, and with only Umbrina xantii found during all events considered.

\section{SEPTENTRIONAL INVADERS AND PARTICULAR EN EVENTS}

The invasion of fish species during EN events has been reported in the literature. Table 6 summarizes this information including original data from 1997/98, 2002/03 and 2004/05.

The effect of EN 1982/83 included 57 invading warmwater species (northern most sampling site) of which $67 \%$ (38) were still detected as far south as Antofagasta $\left(23^{\circ} 40^{\prime} \mathrm{S}\right)$ (Table 6). 67\% (38 species) were epipelagic, 21\% softbottom coastal fishes, and 10.5\% (6 species) rocky-shore fishes (Table 3). The epipelagic species which reached Antofagasta were carangids (8 species, except Naucrates ductor just reaching Iquique), coryphaenids (1 species), scombroids (5 species), sphyraenids (2 species), a nomeioids (1 species) and tetragonurids (1 species). Littoral fishes were principally sandy shore inhabitants (4 sciaenids), 2 balistids and 1 chaetodontid, both rocky shore inhabitants. EN invading sciaenids extended their distribution as far south as Chipana $\left(21^{\circ} 20^{\prime} \mathrm{S}\right)$. 
Table 4. Record of the number and percentage of fish species and families having extended their distribution to northern Chilean waters during EN events between 1983 and 2003. For locations see also Fig. 1 / Registro del número y el porcentaje de especies de peces que extendieron su distribución al norte de Chile durante eventos EN entre 1983 y 2003. Para localidades ver tambien Fig. 1

\begin{tabular}{lcrrrr}
\hline \multirow{2}{*}{ Localities } & \multicolumn{2}{c}{ Location } & sishes & \multicolumn{2}{c}{ Fish families } \\
& Latitude & $\mathrm{N}$ & $\%$ & $\mathrm{~N}$ & $\%$ \\
\hline Arica & $18^{\circ} 21^{\prime} \mathrm{S}$ & 100 & 100 & 52 & 100.0 \\
Pisagua & $19^{\circ} 31^{\prime} \mathrm{S}$ & 90 & 90 & 50 & 96.2 \\
Iquique & $20^{\circ} 12^{\prime} \mathrm{S}$ & 89 & 89 & 48 & 92.3 \\
Punta Gruesa & $20^{\circ} 20^{\prime} \mathrm{S}$ & 69 & 69 & 43 & 82.7 \\
Punta Patache & $20^{\circ} 50^{\prime} \mathrm{S}$ & 68 & 68 & 42 & 80.8 \\
Playa Chipana & $21^{\circ} 20^{\prime} \mathrm{S}$ & 66 & 66 & 42 & 80.8 \\
Tocopilla & $22^{\circ} 05^{\prime} \mathrm{S}$ & 57 & 57 & 38 & 73.1 \\
Mejillones & $23^{\circ} 05^{\prime} \mathrm{S}$ & 56 & 56 & 37 & 71.2 \\
Antofagasta & $23^{\circ} 40^{\prime} \mathrm{S}$ & 53 & 53 & 35 & 67.3 \\
Total & $18^{\circ} 21^{\prime}-23^{\circ} 40^{\prime} \mathrm{S}$ & 100 & 100 & 52 & 100.0 \\
\hline
\end{tabular}

EN 87/88 triggered the southward migration of 16 warmwater fishes, with 56\% epipelagic species and 31\% rockyshore fishes (Table 3). Most of these species were only distributed as far south as Iquique (20'12'S), 44\% until Chipana ( $\left.21^{\circ} 20^{\prime} \mathrm{S}\right)$ and only $6 \%$ south of Tocopilla ( $\left.22^{\circ} 05^{\prime} \mathrm{S}\right)$ (Table 6). Pelagic-oceanic species detected were Zu cristatus, Dasyatis violacea, Auxis rochei, Scomberomorus sierra and Cubiceps carinatus. Pelagic-coastal species (Anchoa naso, Etrumeus teres and Atherinella nocturna) only reached as far south as Chipana. Rocky-shore fishes registered were Bodianus eclancheri and Halichoeres dispilus, detected as far south as Iquique and Punta Gruesa (20²0’S). In sandy-shore habitats only Umbrina xantii was identified as occasional visitor off Chipana.

During EN 91/93, 29 warm-water fishes were detected (Table 3). $76 \%$ were epipelagic, $17 \%$ soft bottom inhabitants and only one species typical for rocky shores (Table 3). EN 97/99 induced strong changes in the ichthyofaunal composition, affecting particularly the epipelagic assemblage. Typical warm-water epipelagic, oceanic species occurred as far south as Antofagasta (2340'S) (Table 2; Dasyatis brevis, Sphyrna zygaena, Strongylura exilis, Fodiator acutus, Sphyraena ensis, Peprilus medius, Mola mola/ramsayi, scombrids: Auxis rochei/thazard and Scomberomorus sierra, carangids: genera Caranx, Decapterus, Oligoplites, Naucrates, Alectis, Selene). Several species were only detected as far south as Iquique (20¹2'S) and Chipana (2120'S) (Naucrates ductor, Dasyatis violacea, Belone stolzmanni, Fistularia petimba,
Table 5. Positive thermal anomalies of EN events and recorded warm-water species off northern Chile (Chile/Peru: Fuenzalida 1992, Fuenzalida et al. 1999, Garcés-Vargas et al. 2005; 5N5-S/120\%W-170oW: Mc Phaden 2003) / Anomalías termicas positivas de los eventos EN a las especies de aguas cálidas registradas frente al norte de Chile (Chile/ Perú: Fuenzalida 1992, Fuenzalida et al. 1999, Garcés-Vargas et al. 2005; 5N-5ㅇ/ 120W-170W: Mc Phaden 2003)

\begin{tabular}{lccccc}
\hline ENSO Events & $82 / 83$ & $87 / 88$ & $91 / 92$ & $97 / 98$ & $02 / 04$ \\
\hline $\mathrm{T}^{\circ}$ anomaly off Chile/Peru & $+5.5^{\circ} \mathrm{C}$ & $+2.5^{\circ} \mathrm{C}$ & $+4.0^{\circ} \mathrm{C}$ & $+5.0^{\circ} \mathrm{C}$ & $+2.0^{\circ} \mathrm{C}$ \\
$\mathrm{T}^{\circ}$ anomaly in the tropical Pacific & $+2.5^{\circ} \mathrm{C}$ & $+1.0^{\circ} \mathrm{C}$ & $+1.5^{\circ} \mathrm{C}$ & & \\
Warm-water fishes off northern Chile & 57 & 16 & 30 & 62 & 5 \\
\hline
\end{tabular}

Scomberesox saurus and Sphyraena idiastes). Typical Peruvian coastal pelagic species were also detected at the bays of Arica (Anchoa nasus), Iquique (A. naso), Chipana (Atherinella noctura) and Antofagasta (Etrumeus teres and Opistonema libertate) (Table 2). The soft-bottom littoral community included Ophicthus zophochir, O. tetratrema, Sciaena starksii, Stellifer minor, Symphurus elongatus, Mulloidichthys dentatus and Arius seemanni at Arica Bay, Bothus constellatus at Caleta Buena, Etropus ectenes and Stellifer erycimba at Iquique, and Umbrina xantii, Eucinostomus argenteus, Pristidactylus approximans, Pseudupeneus grandisquamis and Sphoeroides lobatus/trichocephalus as far south as Antofagasta (Table 2). At the rocky shore, Labrisomus xantii was detected in Caleta Buena; Scorpaena hystrio, Antennarius avalonis, Nicholsina denticulatum and Halichoeres dispilus as far south as Iquique, and Pristigenys serrula, Chaetodon humeralis and Hippocampus ingens off Antofagasta (Table 2).

The weak EN 02/03 triggered the southward migration of only a few species, mainly the pelagic Sphyrna zygaena as far as Antofagasta and Dasyatis violacea as far as Iquique, Caulolatilus princeps down to Pisagua, Balistes polylepis as far as Iquique and Squatina armata was found off Chipana (Table 2).

\section{BIOGEOGRAPHICAL IMPLICATIONS}

The latitudinal distribution of neritic fishes between $0^{\circ} \mathrm{S}$ and $57^{\circ} \mathrm{S}$ (Gulf of Guayaquil to Cape Horn) (Fig. 3) during normal conditions indicate south of the Ecuatorial Front, the existence of three main assemblages (separation under $40 \%$ of similarity): $5^{\circ}-13^{\circ} \mathrm{S}$ (Paita to Pisco), $13-41^{\circ} \mathrm{S}$ (Pisco to Puerto Montt) and $41-57^{\circ} \mathrm{S}$ (Puerto Montt to Cape Horn). The coastal fish assemblages distributed north of the Equatorial Front during normal conditions belong to the Panamic Province that reaches north to the Gulf of California and south to Cabo Blanco (north Peru). Hooker 
Table 6. Number of invading warm-water fishes associated with EN events off northern Chile between 1983 and 2004 / Número de peces invasores de aguas cálidas asociados a eventos EN frente al norte de Chile entre 1983 y 2004

\begin{tabular}{lccccc}
\hline & \multicolumn{5}{c}{ Periods } \\
Localities & $83-85$ & $87-89$ & $91-95$ & $97-00$ & $02-04$ \\
\hline Arica & 57 & 16 & 30 & 62 & 5 \\
Pisagua & 56 & 16 & 29 & 53 & 5 \\
Iquique & 55 & 16 & 29 & 51 & 4 \\
Punta Gruesa & 46 & 10 & 14 & 37 & 2 \\
Patache & 46 & 8 & 13 & 36 & 2 \\
Chipana & 46 & 7 & 13 & 36 & 2 \\
Tocopilla & 42 & 1 & 5 & 33 & 1 \\
Mejillones & 41 & 1 & 4 & 33 & 1 \\
Antofagasta & 38 & 1 & 4 & 33 & 1 \\
& & & & & \\
\hline
\end{tabular}

(2009) discussed the presence of tropical fishes in northern Peru and Hooker (1998) showed the presence of several of these species as far south as Pucusana Bay $\left(12^{\circ} 28^{\prime} \mathrm{S}\right)$ during EN 1997/98.

This distribution pattern is consistent with further biogeographic classification proposed by Balech (1954), López (1963), Lutjeharms (1990), Parin (1991) and Pequeño \& Lamilla (1993) who have suggested a Peruvian, Chilean and Magellan Zooprovince for the southeast Pacific. Pequeño (2000) proposed a different classification, but also related with the before ones, considering a Capricornic Province that includes an Atacaman District and a Central Chilean District, and the Fjord Province including a Chiloé District, and the Fuegian District.

Ojeda et al. (2000) suggested the existence of only two instead of three fish groups with different association: the tropical and subtropical species, corresponding to the Peruvian Province, and subantarctic species, corresponding to the Magallan Zooprovince. The zone between $30^{\circ} \mathrm{S}$ and $42^{\circ} \mathrm{S}$ (central Chile) corresponds to a transition zone inhabited by subantarctic, subtropical, panoceanic and some endemic species as quoted by Mead (1970). This concept is also supported by the main biogeographical features observed by Castilla et al. (1993) and Fernández et al. (2000) separating the Chile-Peru Province, located between Paita and Valparaíso and the Magellan Province between Chiloé and Cape Horn.

In the present classification (see Fig. 3) the $13-41^{\circ} \mathrm{S}$ cluster represents a Chile-Peru Province, including a 13$34^{\circ} \mathrm{S}$ subcluster $\left(13-21^{\circ} \mathrm{S}\right.$ and $21-34^{\circ} \mathrm{S}$ with 0.80 similarity). The $34-41^{\circ} \mathrm{S}$ zone represents the south part of the province and can not be regarded as a transition zone into the Magellan Province $\left(41-57^{\circ} \mathrm{S}\right)$ because of their low similarity level (20\%).

The differences between Ojeda et al. (2000) and the present results may be explained because of numerous Panamic and south Peruvian warm-water species, were not considered by the first author.

\section{Discussion}

The warm EN and the cold LN phases of the southern Pacific Oceans sea surface temperature induce a latitudinal displacement of the fauna, for which two categories of migrating species were proposed: The "stenoterm fishes of warm waters" comprehend fishes that regularly inhabit subtropical waters associated to the Chilean oceanic islands, while "septentrional invaders of the Peru Current" are migrants to southern latitudes (Mann 1954), of panamic origin, invading the Peruvian and Chilean coast, and Peruvian species that migrate to northern Chile during EN events.

The southern distribution limit of these warm-water fishes during EN is related with the intensity of the positive temperature anomaly of the events analysed. In many cases, and especially in the case of coastal species (sciaenids, ariids, labrids, ophichthids, bothids), Península de Mejillones represents a natural barrier for the southerly migration of several species, proposed by Escribano et al. (2002) and Castilla et al. (2002).

Epipelagic fishes (scombrids, carangids, centrolophids, exocoetids) seem to react more rapidly to EN conditions (see Tables 2 and 3) compared to the more resilient littoral fishes. Oceanic fishes probably migrate off-shore from southern Peru along the temperature gradient of subtropical waters described by Schweigger (1960) and Blanco et al. (2001), west of the coastal upwelling cells off northern Chile. In contrast the southerly migration of rocky-shore fishes is slow because they are not that mobile and in many cases with territorial behaviour like clinids and blennids. The specimens caught after EN conditions are normally fully developed and can be detected for a much longer period revealing a time lack compared to oceanographic conditions. For example Halichoeres dispilus, Ophicthus tetratrema, Antennarius avalonis, Nicholsina denticulatus and Arius semanni (Table 1) were captured in 2000/2001 during LN conditions, 3 years after EN 97/98. They probably are not able to reproduce successfully due to unfavourable environmental conditions. 


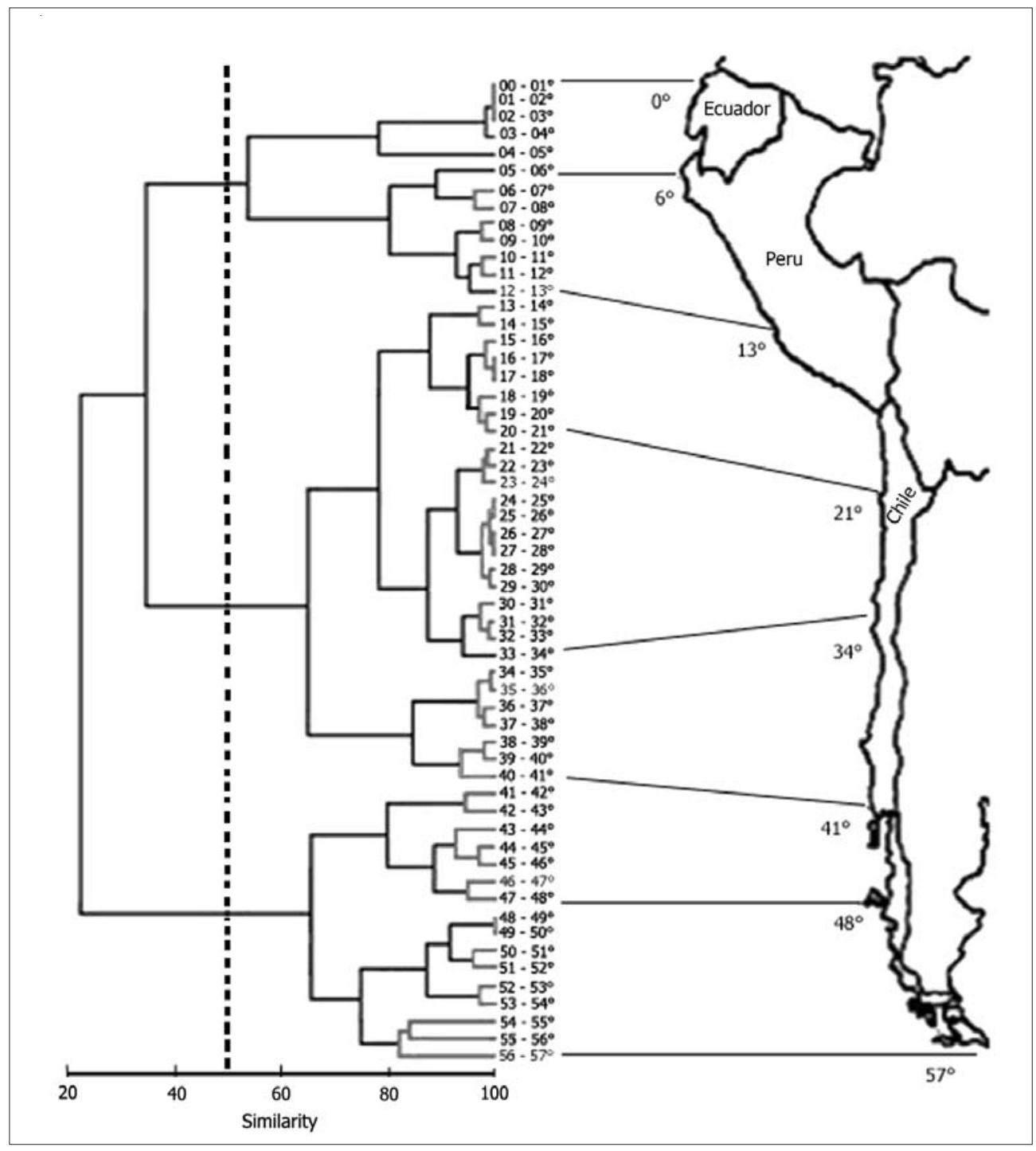

Figure 3. Sorensen similarity dendrogramm of neritic fish assemblages using the hierarchical cluster analysis routine CLUSTER in the program PRIMER 6 and SIMPROF permutation test (at $1 \%$ level) to determine significant internal structure of the clusters / Dendrograma de similitud de Sorensen de los ensambles de los peces neríticos utilizando la rutina de analisis jerárquico de agrupaciones CLUSTER del programa PRIMER 6 y el test de permutación SIMPROF (al nivel del 1\%) para la determinación de estructuras internas significativas de las agrupaciones

From a ichthyogeographical point of view, inside the 13$41^{\circ} \mathrm{S}$ cluster (Pisco/Puerto Montt) (Fig. 3) the southward oscillations of the normal $20-21^{\circ}$ zoogeographic border (Pisco-Chipana cluster) south to $24^{\circ} \mathrm{S}$ (south of Antofagasta) during strong ENSO (1982/83 and 1997/98) (Fig. 4), is very consistent with the oceanographic changes associated to ENSO (Fig. 5). Otherwise if a northward oscillations of the $21-34^{\circ} \mathrm{S}$ cluster during strong La Niña may be expected, than the the area investigated during this study $\left(18^{\circ}-21^{\circ} \mathrm{S}\right)$ may be regarded as a transition zone between the central Chilean and the Peruvian faunistic zones, with north/south oscillations following to the respective thermal anomalies and oceanographic patterns linked to EN events.

\section{Acknowledgments}

In memory of Ismael Kong we express our recognition for his enormous contributions to knowledge of biology and systematic of the fishes of northern Chile. His work is an unquestionably legacy for the coming generations of ichthyologists of the future. We also remember our friend Mario Villegas, colleague of many meetings, scientific discussions and field trips, who deceased in March 2008, during the development of the EU Project CENSOR. Finally our thanks to the EU Project CENSOR (Climate Variability and El Niño Southern Oscillation: Implications for Natural Resources and Managament, contract 511071) and the Programa Bicentenario de Ciencia y Tecnología CONICYTCENSOR-RUE02, which funded this study. 


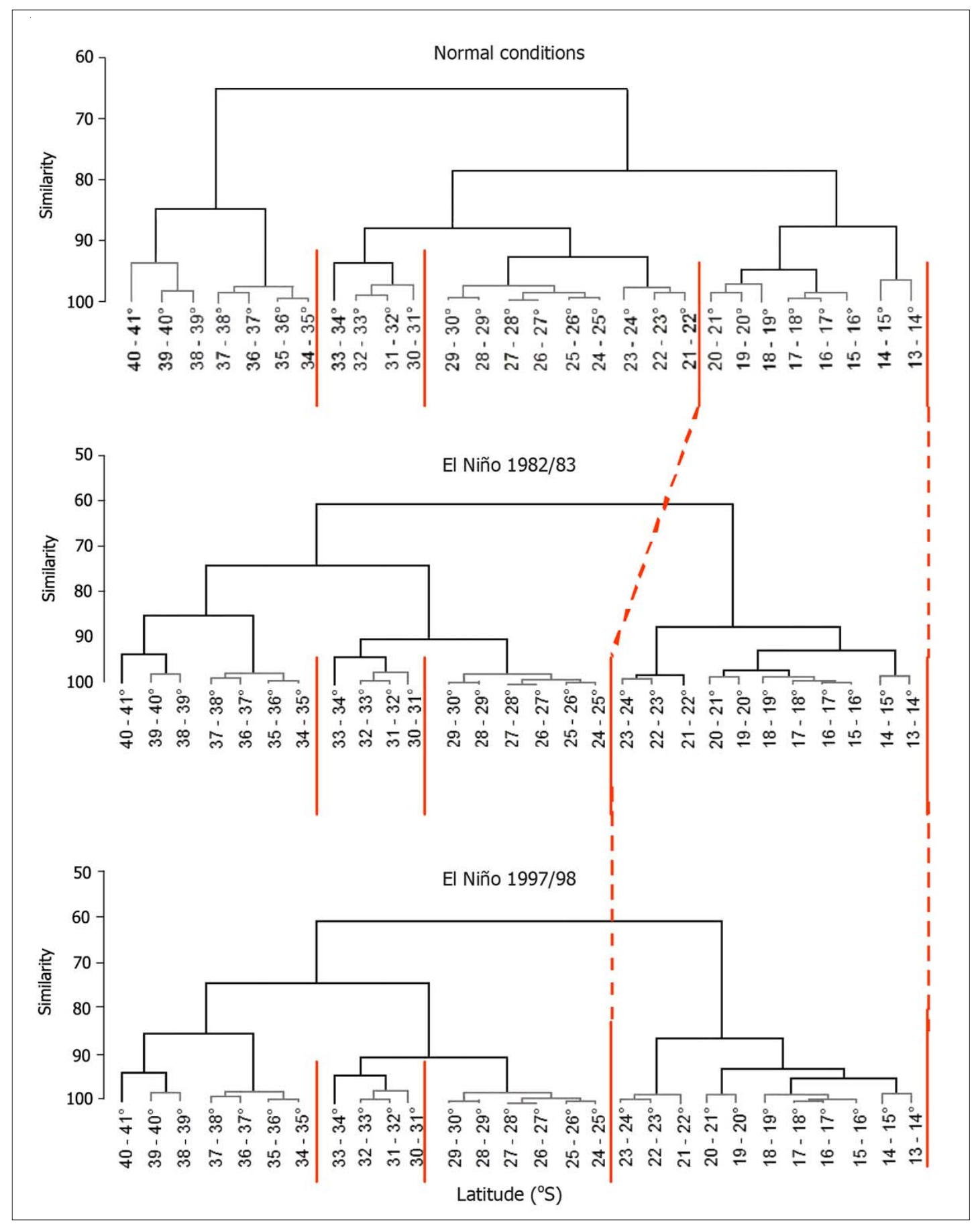

Figure 4. Sorensen similarity dendrogramm of neritic fish assemblages during normal conditions, El Niño 1982/83 and 1997/98 using the hierarchical cluster analysis routine CLUSTER in the program PRIMER 6 and SIMPROF permutation test (at $1 \%$ level) to determine significant internal structure of the clusters / Dendrograma de similitud de Sorensen de las agrupaciones de los peces neríticos durante condiciones normales, El Niño 1982/ 83 y 1997/ 98 utilizando la rutina de analisis jerárquico de agrupaciones CLUSTER del programa PRIMER 6 y el test de permutación SIMPROF (al nivel del 1\%) para la determinación de estructuras internas significativas de las agrupaciones 


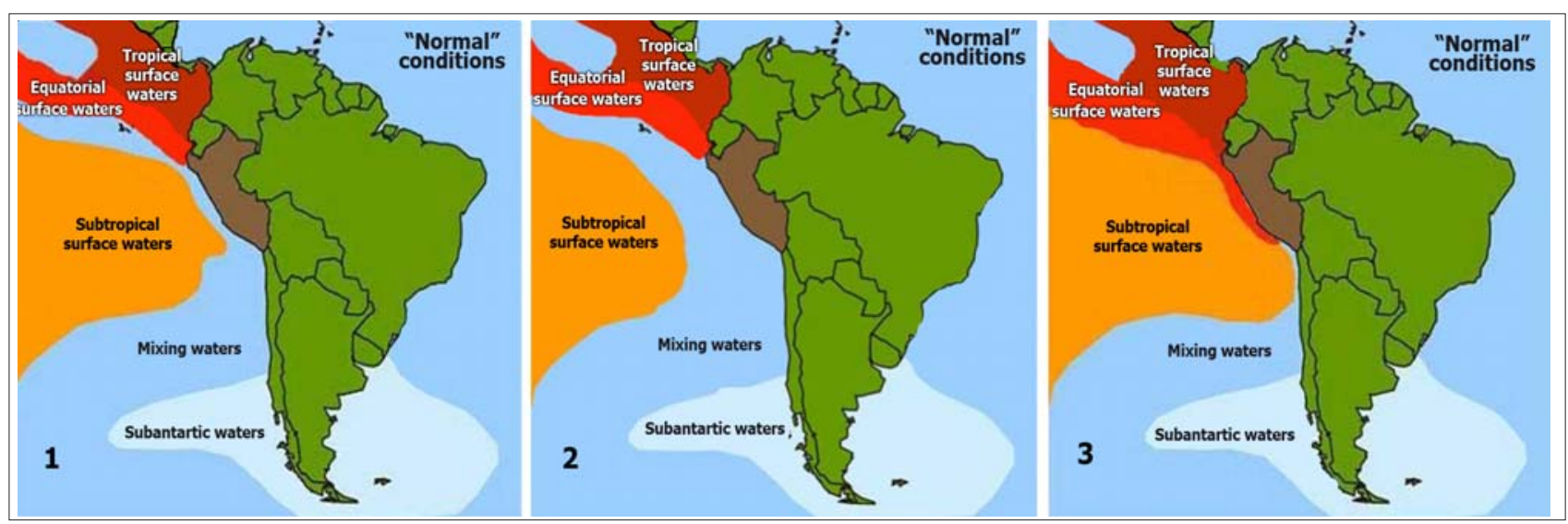

Figure 5. Schematic representation of the principal water masses of the southeast Pacific during 1) normal conditions 2) cold La Niña conditions and 3) warm El Niño conditions. (fide Keyl et al. 20086) / Representación esquemática de las principales masas de agua del Pacífico suroriental durante 1) condiciones normales, 2) condiciones frías La Niña y 3) condiciones cálidas El Niño. (fide Keyl et al. 20086)

\section{LITERATURE CITED}

Alarcón E. 1975. Oceanographic conditions in coastal waters off Coquimbo. International Symposium Coastal Upwelling Proceedings, Coquimbo, Chile, November 18-19, Fundación Chile, pp. 149-161.

Alberti V. 1963. Primera descripción para Chile de un ejemplar de Pseudopriacanthus. Investigaciones Zoológicas Chilenas 10: 101-105.

Arntz W. 1986. The two faces of El Niño 1982-83. Meeresforschung. Report on Marine Research 31: 1-46.

Bahamonde N. 1963. Pez luna (Mola ramsayi) en Chiloé. Noticiario Mensual, Museo Nacional de Historia Natural 82: 4-8.

Bahamonde N \& G Pequeño. 1975. Peces de Chile. Lista Sistemática. Publicación Ocasional, Museo Nacional de Historia Natural 22: 3-20.

Balech E. 1954. División zoogeográfica del litoral sudamericano. Revista de Biología Marina 4: 184-195.

Barber RT \& FP Chavez. 1983. Biological consequences of El Niño. Science 222: 1203-1210.

Barber RT \& RL Smith. 1981. Coastal upwelling ecosystems. In: Longhurst AR (ed). Analysis of marine ecosystem, pp. 31-68. Academic Press, New York.

Bernal P, F Robles \& O Rojas. 1983. Variabilidad física y biológica en la región meridional del sitema de corrientes ChilePerú. FAO Informe de Pesca 291(3): 682-711.

Berrios V \& M Vargas. 2000. Estructura del ensamble de peces intermareales de la costa rocosa del norte de Chile. Revista de Biología Marina y Oceanografía 35(1): 73-81.

Berrios V \& M Vargas. 2004. Estructura trófica de la asociación de peces intermareales de la costa del norte de Chile. Revista de Biología Tropical 52(1): 201-212.

Bini G. 1952. Osservazioni sulla fauna marina delle coste del Chile e del Peru con speciale riguardo alle specie ittiche in

${ }^{6}$ Keyl F, J Argüelles \& R Tafur. 2008. On the population structure, age and growth of Dosidicus gigas in the Peruvian EEZ. Centre for Tropical Marine Ecology \& Instituto del Mar del Perú. Proyecto EU / CENSOR generale ed ai tonni particolare. Bollettino di Pesca, Piscicultura e Idrobiologia 7(1): 11-60.

Blanco J, AC Thomas, ME Carr \& PT Strub. 2001. Seasonal climatology of hydrographic conditions in the upwelling region off northern Chile. Journal of Geophysical Research 106(C6): 11.451-11.467.

Burkov VA, SG Panfilova, LK Moiseev \& AB Zubin. 1971. Circulation and water masses of the South-Eastern Pacific. Trudy Instituta Okeanology 89: 9-32.

Castilla JC, NA Lagos, R Guiñez \& JL Largier. 2002. Embayments and nearshore retention of plankton: The Antofagasta Bay and other Examples. In: Castilla JC \& JL Largier (eds). The oceanography and ecology of the nearshore and bays in Chile, pp. 179-203. Ediciones Universidad Católica de Chile, Santiago.

Chirichigno N. 1974. Clave para identificar los peces marinos del Perú. Informe, Instituto del Mar del Perú 44: 1-387.

Chirichigno N \& J Vélez. 1998. Clave para identificar los peces marinos del Perú, 496 pp. Publicación Especial, Instituto del Mar del Perú, Callao.

Clarke KR \& RN Gorley. 2006. Primer V6: user manual/tutorial, 192 pp. PRIMER-E, Plymouth.

Cushing DJ. 1971. Upwelling and the production of fish. Advances in Marine Biology 9: 255-334.

De Buen F. 1953. Contribución a la ictiología IV. Sobre la presencia del atún (Thunnus thynus) en la costa de Chile Central. Investigaciones Zoológicas Chilenas 1(10): 3-4.

De Buen F. 1957. Los peces de la familia Molidae. Investigaciones Zoológicas Chilenas 4: 66-76.

De Buen F. 1959a. Presencia en aguas de Chile de una rara especie de rémora Echeneis (Remilegia) australis. Investigaciones Zoológicas Chilenas 4: 55-64.

De Buen F. 1959b. Lampreas, tiburones, rayas y peces de la Estación de Biología Marina de Montemar, Chile. Revista de Biología Marina 9(1-3): 1-200. 
De Buen F. 1959c. Notas preliminares sobre la fauna marina preabismal de Chile, con descripción de una familia de rayas, dos géneros y siete especies nuevas. Boletín del Museo Nacional de Historia Natural 27(3): 173-201.

Delfín FT. 1899. Catálogo de los peces de Chile. Revista Chilena de Historia Natural 3: 24, 57-62, 79-85, 95-99, 110-113, 121-124, 131-134, 153-161.

Delfín FT. 1900. Catálogo de los peces de Chile. Revista Chilena de Historia Natural 4: 4-6, 21-25, 29-31, 55-58, 69-71, 8284, 110-111, 129-131, 166-167, 185-186.

Delfín FT. 1901. Ictiolojía chilena. Catálogo de los peces de Chile. Revista Chilena de Historia Natural 3: 7-133.

Delfín FT. 1903. Adición al ‘Catálogo de los peces de Chile’ con descripción de una nueva especie. Revista Chilena de Historia Natural 7: 220-225.

Escribano R, VH Marin, P Hidalgo \& G Olivares. 2002. Physical-biological interactions in the pelagic ecosystem of the nearshore zone of the Northern Humboldt Current System. In: Castilla JC \& JL Largier (eds). The oceanography and ecology of the nearshore and bays in Chile, pp. 145-179. Ediciones Universidad Católica de Chile, Santiago.

Fernández M, E Jaramillo, P Marquet, C Moreno, S Navarrete, P Ojeda, C Valdovinos \& J Vasquez. 2000. Diversity, dynamics and biogeography of Chilean benthic nearshore ecosystems: an overview and guidelines for conservation. Revista Chilena de Historia Natural 73: 797830.

Figueroa D. 2002. Forcing of physical exchange in the nearshore Chilean Ocean. In: Castilla JC \& JL Largier (eds). The oceanography and ecology of the nearshore and bays in Chile, pp. 31-43. Ediciones Universidad Católica de Chile, Santiago.

Fitch J. 1951. Studies and notes on some California marine fishes. Report California Department of Fish and Game 37(2): 111-120.

Fowler H. 1945. Fishes of Chile. Systematic catalog. Apartado de la Revista Chilena de Historia Natural 1941-1943 (45-47): 1-36, 1-171.

Fuenzalida R. 1992. Anomalías oceanográficas y meteorológicas durante el desarrollo de El Niño 1991-92 en la zona de Iquique (20¹8’S). Investigación Pesquera 37: 67-72.

Fuenzalida R, J Blanco, R Nuñez \& S Hormazabal. 1999. Oceanographic and meteorological effects during the El Niño event 1997-98, at the Chilean coast $\left(20^{\circ} \mathrm{S}\right)$. Revista de Investigaciones Científicas y Tecnológicas. Serie Ciencias del Mar 4: 1-3.

Fuenzalida R, W Schneider, J Garcés-Vargas, L Bravo \& C Lange. 2009. Vertical and horizontal extension of the oxygen minimum zone in the eastern South Pacific Ocean. Deep-Sea Research II 56: 992-1003.

Garcés-Vargas J, W Schneider, R Abarca del Río, R Martinez \& E Zambrano. 2005. Inter-annual variability in the thermal structure of an oceanic time series station off Ecuador (19902003) associated with El Niño events. Deep Sea Research I 52(10): 1789-1805.
Guichenot A. 1848. Peces. In: Gay C (ed). Historia física y política de Chile. Zoología 2: 137-370. Claudio Gay \& Museo de Historia Natural de Santiago, Paris / Santiago de Chile.

Guzmán G, M Vargas \& W Sielfeld. 1998. Colección de peces en el museo del mar y sala de colecciones de la Universidad Arturo Prat (Iquique, Chile). Revista de Investigaciones Científicas y Tecnológicas. Serie Ciencias del Mar 4: 9-30.

Hildebrand SF. 1946. A descriptive catalog of the shore fishes of Perú. Bulletin of the United States National Museum 189: 1-530.

Hooker Y. 1998. Fauna asociada a las aguas cálidas presentes en la bahía Pucusana durante El Niño 1997/98: Junio a Julio 1997. Informes Progresivos, Instituto del Mar del Perú 85: 120

Hooker Y. 2009. Nuevos registros de peces costeros tropicales para el Perú. Revista Peruana de Biología 16(1): 33-41.

Hormazabal S \& G Shaffer. 2002. Tropical Pacific control of intraseasonal osscillations off Chile by way of oceanic and atmospheric pathways. Geophysical Research Letters 29(6): 1-4.

Hormazabal S, G Shaffer, J Letelier \& O Ulloa. 2001. Local and remote forcing of sea surface temperature in the coastal upwelling system off Chile. Journal of Geophysical Research 106: 16.657-16.671.

Hoyos L, J Tarazona, B Shiga \& V Chiong. 1985. Algunos cambios en la ictiofauna y sus relaciones tróficas durante el fenómeno "El Niño" en la bahía de Ancón. In: Arntz WE, A Landa \& J Tarazona (eds). El Fenómeno El Niño. Su impacto en la fauna marina. Boletín, Instituto del Mar Perú Vol. Extraordinario: 163-171.

Jordan DS \& CH Eigenmann. 1890. A review of the genera and species of Serranidae found in the waters of America and Europe. Bulletin of the United States Fish Commission 8: 329-441.

Jordan DS \& BW Evermann. 1896. The fishes of North and Middle America: a descriptive catalogue of the species of fish-like vertebrates found in the waters of North America, north of the Isthmus of Panamá. Part I. Bulletin of the United States National Museum 47: 1-1240.

Jordan DS \& BW Evermann. 1898. The fishes of North and Middle America: a descriptive catalogue of the species of fish-like vertebrates found in the waters of North America, north of the Isthmus of Panamá. Part II. Bulletin of the United States National Museum 47: 1241-2183.

Jordan DS \& BW Evermann. 1900. The fishes of North and Middle America: a descriptive catalogue of the species of fish-like vertebrates found in the waters of North America, north of the Isthmus of Panamá. Part IV. Bulletin of the United States National Museum 47: 3137-3313.

Kong I \& A Bolados. 1987. Sinopsis de peces asociados al fenómeno "El Niño" 1982-83 en el norte de Chile. Estudios Oceanológicos 6: 25-58.

Kong I \& J Valdés. 1990. Sciaenidos de Chile: Análisis taxonómico y morfológico. Estudios Oceanológicos 9: 13-56. 
Kong I, J Tomicic \& C Guerra. 1981. Prystigenys serrula (Gilbert) en la Bahía de San Jorge (Antofagasta, Chile) Pisces, Priacanthidae. Noticiario Mensual, Museo Nacional de Historia Natural 298: 9-14.

Kong I, J Tomicic \& J Zegers. 1985. Ictiofauna asociada al fenómeno El Niño 1982-83 en la zona norte de Chile. Investigación Pesquera 32: 215-224.

Kong I, M Oliva \& L Marinovic. 1999. Antecedentes sobre la ictiofauna trópico ecuatorial recolectada en el norte de Chile. Seminario-Taller Impacto de los eventos "El Niño Oscilación del Sur" sobre la diversidad Biológica de America latina, LimaPerú, p. 41.

Kong I, M Oliva \& L Marinovic. 2002. Antecedentes sobre la ictiofauna trópico-ecuatorial recolectados en el norte de Chile. Informe Técnico, Universidad de Antofagasta, Antofagasta, pp. 1-5.

López R. 1963. Problemas sobre la distribución geográfica de los peces marinos sudamericanos. Revista del Museo Argentino de Ciencias Naturales, Hidrobiología 1(3): 111-135.

Lutjeharms JRE. 1990. The oceanography and fish distribution of the Southern Ocean. In: Gon O \& PC Heemstra (eds). Fishes of the Southern Ocean, pp. 6-27. JLB Smith Institute of Ichthyology, Grahamstown.

Mann G. 1954. La vida de los peces en aguas chilenas, 342 pp. Instituto de Investigaciones Vetererinarias, Universidad de Chile, Santiago.

Martinez C, C Zalazar, G Böhm, J Mendieta \& C Estrada. 1985. Efectos del fenómeno El Niño 1982-83 sobre los principales recursos pelágicos y su pesquería (AricaAntofagasta). Investigación Pesquera 32: 129-139.

McPhaden MJ. 2003. Tropical Pacific Ocean heat control variations and ENSO persistence barriers. Geophysical Research Letters 30(9): 1480, <doi: 10.1029-2003 GLO 16872>

Mead GW. 1970. A history of South Pacific fishes. In: Wooster WS (ed). Scientific exploration of the South Pacific, pp. 236251. National Academy of Sciences, Washington.

Morales CE, J Blanco, M Braun, H Reyes \& N Silva. 1996. Chlorophyll-a distribution and associated oceanographic conditions in the upwelling region off northern Chile during the winter spring 1993. Deep Sea Research A 43: 267-289.

Morrow J. 1957. Shore and pelagic fishes from Perú, with new records and the description of a new species of Sphoeroides. Bulletin of the Bingham Oceanographic Collection 16(2): 555.

Oliver-Schneider C. 1930. Algunas observaciones sobre el pez luna (Mola mola Lin.) Revista Chilena de Historia Natural 34: 200-207.

Oliver-Schneider C. 1943a. Catálogo de los peces marinos del litoral de Concepción y Arauco. Levantamiento biológico de la Provincia de Concepción, 59 pp. Litografía Concepción SA, Concepción.

Oliver-Schneider C. 1943b. Catálogo de los peces marinos del litoral de Concepción y Arauco. Boletín de la Sociedad de Biología de Concepción 17: 75-126.
Ojeda P. 1978. Nuevos registros para la ictiofauna de Arica, Chile (Pisces, Teleostomi). Noticiario Mensual, Museo Nacional de Historia Natural 261: 7-9.

Ojeda P, F Labra \& A Muñoz. 2000. Biogeographic patterns of Chilean littoral fishes. Revista Chilena de Historia Natural 73: 625-641.

Parin NV. 1991. Fish fauna of the Nazca and Sala y Gomez submarine ridges, the easternmost outpost of the indo-west Pacific zoogeographic region. Bulletin of Marine Science 49(3): 671-683.

Pequeño G. 1978. Doce nuevos registros para la costa de Valdivia, Chile, y su alcance ictiogeográfico. Revista de la Comisión Permanente del Pacífico Sur 9: 109-126.

Pequeño G. 1989. Peces de Chile. Lista sistemática revisada y comentada. Revista de Biología Marina 21(2): 1-132.

Pequeño G. 1997. Fishes of Chile. Reviewed and annotated checklist: Addendum. Revista de Biología Marina y Oceanografía 32: 77-94.

Pequeño G. 2000. Delimitaciones y relaciones biogeográficas de los peces del océano Pacífico sur oriental. Estudios Oceanológicos 19: 53-76.

Pequeño G \& J Lamilla. 1995. Batoideos comunes a las costas de Chile y Argentina-Uruguay (Pisces: Chondrichthyes). Revista de Biología Marina 28(2): 203-217.

Philippi R. 1876. Descripción de tres peces nuevos. Anales de la Universidad de Chile 48: 261-266.

Philippi R. 1887. Historia natural. Sobre los tiburones y algunos otros peces de Chile. Anales de la Universidad de Chile 71: 342.

Philippi R. 1892. Algunos peces de Chile. Las rayas, Callorhynchus y Orthagoriscus chilenos. Anales del Museo Nacional de Chile, Zoología 9: 1-16.

Quinn W, D Zopf, K Short \& R Kuo-Yang. 1978. Historical trends and statistics of the Southern Oscillation, El Niño, and Indonesian droughts. Fishery Bulletin 76(3): 663-678.

Radovich J. 1961. Relationship of some marine organism of the northeast Pacific to water temperatures particulary during 1957 through 1959. California Department of Fish \& Game, Fish Bulletin 112: 1-62.

Robles F, E Alarcón \& A Ulloa. 1976. Las masas de agua en la región norte de Chile y sus variaciones en un período frío (1967) y en períodos cálidos (1969, 1971-73). Reunión de trabajo sobre el fenómeno conocido como "El Niño". Guayaquil-Ecuador, 4-12 de diciembre 1974. FAO Informe de Pesca 185: 94-196.

Rojas J \& G Pequeño. 2001. Revisión taxonómica de especies de las subfamilias Epinephelinae y Serraninae (Pisces: Serranidae) de Chile. Revista de Biología Tropical 49(1): 155169.

Rutland J. 1982. Variabilidad diaria, estacional e interanual de la densidad de potencia eólica teórica en la costa norte de Chile. Tralka 2: 161-176.

Ryther JH. 1969. Photosynthesis and fish production in the sea. Science 166: 72-76. 
Schweigger E. 1960. Fenómenos hidrográficos y biológicos en el sur del Perú y en el norte de Chile. Revista de Biología Marina 10: 51-67.

Sielfeld W \& M Vargas. 1996. Composición y estructura de la ictiofauna demersal en la zona norte de Chile. Investigaciones Marinas 24: 3-17.

Sielfeld W \& M Vargas. 1999. Review of marine zoogeography of Chilean Patagonia $\left(42^{\circ}-57^{\circ} \mathrm{S}\right)$. Scientia Marina 63(Supl.1): 451-463.

Sielfeld W \& M Vargas. 2000. Primer registro para Chile continental de los peces Scorpaena histrio (Scorpaenidae) y Chilomycterus reticulatus (Diodontidae). Noticiario Mensual, Museo Nacional de Historia Natural 343: 33-38.

Sielfeld W, M Vargas, I Kong \& G Guzmán. 1995. Peprilus medius (Peters, 1869), en Punta Chipana (21ํ19’S), I Región, Chile (Pisces: Perciformes, Stromateidae). Ciencia y Tecnología del Mar 18: 25-31.

Sielfeld W, M Vargas, V Berrios \& G Aguirre. 2002. Warm ENSO Events and their effects on the coastal fish fauna of Northern Chile. Extended Abstracts of the El Niño Symposium \& Workshop. Investigaciones Marinas 30(1): 122-124.

Sielfeld W, M Vargas \& I Kong. 2003. Primer registro de Etropus ectenes Jordan, 1889, Bothus constellatus Jordan \& Goss, 1889, Achirus klunzingeri (Steindachner, 1880) y Symphurus elongatus (Günther, 1868)(Pisces, Pleuronectiformes) en Chile, con comentarios sobre la distribución de los lenguados chilenos. Investigaciones Marinas 31(1): 51-65.

Silva N \& S Neshyba. 1979. On the southernmost extension of the Peru-Chile Undercurrent. Deep-Sea Research A 26: 13871393.

Smith RL. 1992. Coastal upwelling in the modern ocean. In: Summerhayes CP, WL Prell \& KC Emeis (eds). Upwelling systems: evolution since the early Miocene. Geological Society Special Publication 64: 9-28.

Steindachner F. 1898-1913. Die fische der Sammlung Plate. Fauna Chilensis L. Plate 1: 281-338. [Zoologisches Jahrbuch Jena 3: 201-214; 4: 281-338; 6: 201-214].

Tomicic J. 1985. Efectos del fenómeno El Niño 1982-83 en las comunidades litorales de la península de Mejillones. Investigación Pesquera, Chile 32: 209-213.
Varas E \& P Ojeda. 1990. Intertidal fish assemblages of the central Chilean coast: diversity, abundance and trophic patterns. Revista de Biología Marina 25: 59-70.

Vargas L \& G Pequeño. 2004. El estatus taxonómico de Graus fernandezianus Philippi, 1887: nuevo registro geografico y comentarios sobre Graus nigra Philippi, 1887 (Osteichthyes: Perciformes), en Chile. Gayana 68(1): 63-69.

Vargas M. 1993. Estructura comunitaria de la ictiofauna de un área somera asociada a focos de surgencia en el norte de Chile

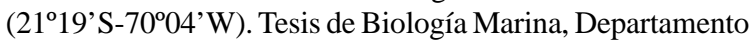
de Ciencias del Mar, Universidad Arturo Prat, Iquique, 215 pp.

Vargas M \& W Sielfeld. 1992. Bathyraja peruana McEachran \& Miyake, 1984 nuevo registro para la condrictiofauna chilena. Revista de Investigaciones Científicas y Tecnológicas. Serie Ciencias del Mar 2: 105-110.

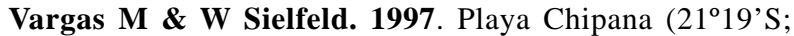
$70^{\circ} 04^{\prime} \mathrm{W}$ ): A nursery and smolting area for marine coastal fish in northern Chile. Archive of Fisheries and Marine Research 45(2): 167-182.

Vargas M \& W Sielfeld. 1999. Caranx caninus (Günther, 1869) y Naucrates ductor (Linnaeus, 1758) nuevos registros de peces Carangidae frente a las costas de Iquique (Chile: I Región). Revista de Investigaciones Científicas y Tecnológicas. Serie Ciencias del Mar 4: 76-81.

Vargas M, W Sielfeld \& N Amado. 1998. Nuevos antecedentes sobre peces Labridae (Osteichthyes, Perciformes) de Chile continental. Boletín de la Sociedad de Biología de Concepción 69: 191-202.

Vargas M, S Cifuentes \& E Emparanza. 1999. Espectro trófico de peces concurrentes al área de crianza Playa Chipana ( $21^{\circ} 19^{\prime} \mathrm{S}-70^{\circ} 04^{\prime} \mathrm{W}$ ) del norte de Chile. Revista de Biología Tropical 47(3): 597-600.

Vasquez JA \& JM Alonso-Vega. 2004. El Niño 1997-98 en el norte de Chile: efectos en la estructura y en la organización de comunidades submareales dominadas por algas pardas. In: Avaria S, J Carrasco, J Ruttland \& E Yañez (eds). El Niño-La Niña 1997-2000. Sus efectos en Chile, pp. 119-135. Comité Oceanográfico Nacional, Valparaíso.

Vélez J, J Zeballos \& M Méndez. 1984. Effects of 1982-83 El Niño on fishes and crustaceans off Peru. Tropical OceanAtmosphere Newsletter 28: 10-12.

Recibido el 20 de julio de 2009 y aceptado el 29 de mayo de 2010 